\title{
Opportunistic Spectrum Sharing Based on OFDM With Index Modulation
}

\author{
Qiang Li, Miaowen Wen, Senior Member, IEEE, Shuping Dang, Member, IEEE, \\ Ertugrul Basar, Senior Member, IEEE, H. Vincent Poor, Fellow, IEEE, and Fangjiong Chen, Member, IEEE
}

\begin{abstract}
In this paper, a novel opportunistic spectrum sharing scheme, based on orthogonal frequency division multiplexing with index modulation (OFDM-IM), is proposed for cognitive radio $(C R)$ networks. In the considered OFDM-IM based CR (OFDM-IM-CR) model, the primary transmitter (PT) communicates with the primary receiver with the aid of an amplifiedand-forward (AF) relay by transmitting OFDM-IM signals. Meanwhile, the secondary transmitter (ST) passively senses the spectrum and transmits its own information over those inactive subcarriers of the primary network to the secondary receiver if the signal-to-noise ratio of the $\mathbf{P T} \rightarrow \mathrm{ST}$ link is above a predefined threshold; otherwise, the ST stays in silent mode. Two different types of maximum-likelihood (ML) detectors are designed for the primary network, based on the knowledge of either the estimated channel state information or the statistical channel information of the secondary network. A complexity-reducing method, which is applicable to both types and achieves near optimal performance, is further proposed. To evaluate the performance, a tight upper bound on the bit error rate (BER) is derived, assuming the first type of ML detection. Simulation results corroborate the analysis and show that OFDM-IM-CR has the potential of outperforming OFDM-CR and OFDM-IM-AF in terms of BER with higher spectral efficiency.
\end{abstract}

Index Terms-Amplify-and-forward relaying, cognitive radio, index modulation, OFDM, spectrum sharing.

Manuscript received February 12, 2019; revised July 14, 2019 and September 11, 2019; accepted September 18, 2019. Date of publication XXXX XX, 2019; date of current version XXXX XX, 2019. This work was supported in part by the National Natural Science Foundation of China under Grant 61871190, Grant 61671211, and Grant U1701265, in part by the Natural Science Foundation of Guangdong Province under Grant 2018B030306005, Grant 2016A030311024, and Grant 2016A030308006, in part by the State Key Laboratory of Integrated Service Networks of Xidian University under Grant ISN20-16, in part by the Pearl River Nova Program of Guangzhou under Grant 201806010171, and in part by the China Scholarship Council under Grant 201806150074. The associate editor coordinating the review of this paper and approving it for publication was G. Alexandropoulos. (Corresponding author: Miaowen Wen.)

Q. Li and M. Wen are with the School of Electronic and Information Engineering, South China University of Technology, Guangzhou 510640, China, and also with the State Key Laboratory of Integrated Service Networks, Xidian University, Xi'an 710071, China (e-mail: eeqiangli@ mail.scut.edu.cn; eemwwen@scut.edu.cn).

S. Dang is with the Computer, Electrical and Mathematical Science and Engineering Division, King Abdullah University of Science and Technology, Thuwal 23955-6900, Kingdom of Saudi Arabia (e-mail: shuping.dang@kaust.edu.sa).

E. Basar is with the Communications Research and Innovation Laboratory, Department of Electrical and Electronics Engineering, Koç University, Sariyer 34450, Istanbul, Turkey (e-mail: ebasar@ku.edu.tr).

H. V. Poor is with the Department of Electrical Engineering, Princeton University, Princeton, NJ 08544, USA (e-mail: poor@princeton.edu).

F. Chen is with the School of Electronic and Information Engineering, South China University of Technology, Guangzhou 510640, China (e-mail: eefjchen@scut.edu.cn).

Digital Object Identifier XXXX

\section{INTRODUCTION}

$\mathbf{T}$ HE radio spectrum is a precious natural resource, and it is traditionally allocated by governments to specific licensees for exclusive use. Although this simple spectrum allocation approach limits the interference between different wireless systems, it leads to two contradictory consequences. On one hand, the remaining unallocated frequency bands become increasingly scarce. On the other hand, there are still substantial unused resources in frequency, time, and space of the licensed spectrum [1]. The latter has motivated the introduction of cognitive radio (CR) [2], which allows unlicensed (secondary) systems to opportunistically use the idle spectrum owned by licensed (primary) systems, improving spectrum utilization significantly. In CR networks, secondary users (SUs) are intelligent devices, which are able to sense their surrounding electromagnetic environments and dynamically adjust certain radio operating parameters for the access to the spectrum of primary users (PUs) [3]. In the literature, there are three main spectrum access strategies: underlay, overlay, and interweave. More specifically, in underlay models, SUs can access the licensed spectrum concurrently used by the primary network if the interference from SUs to PUs is below a tolerable level [4]. In overlay paradigms, SUs achieve spectrum sharing at the cost of sacrificing part of their power to facilitate the primary transmission by cooperative amplifyand-forward (AF) or decode-and-forward (DF) relaying [5]. By contrast, for the interweave strategy, SUs exploit spectrum holes, which are spectrum resources assigned to a PU but not being occupied by that user, for transmission [1]. Since SUs need to continuously detect whether the spectrum is available or not for opportunistic transmission, spectrum sensing is indispensable at SUs and it has been extensively investigated in the literature [6]-[8].

Orthogonal frequency division multiplexing (OFDM) is an easy-to-implement and effective multi-carrier transmission technique, and it has been used in many current wireless communications systems, including IEEE $802.11 \mathrm{a} / \mathrm{g} / \mathrm{n}$ wireless local area networks, Long Term Evolution (LTE), and digital television (DTV) systems. Moreover, OFDM has been recognized as one of the key physical-layer technologies for fifth generation wireless networks. As explained in [9] and [10], OFDM is also an appealing modulation candidate for CR systems due to its inherent sensing and spectrum shaping capabilities. It should be noted that OFDM has already been adopted by the IEEE $802.22 \mathrm{CR}$ standard for reusing the spectrum allocated for TV channels. Therefore, various aspects 
of OFDM-based CR (OFDM-CR) networks, ranging from resource allocation to spectrum monitoring, have received considerable research attention [11]-[15].

Recently, a promising variant of OFDM has been proposed by applying the concept of index modulation (IM) [16]. The rationale behind IM is to utilize the index(es) of some building blocks of a communication system to transmit extra information [17]-[21]. To be more specific, in OFDM with IM (OFDM-IM), only a subset of all available subcarriers are activated to carry ordinarily modulated $M$-ary symbols and the resulting subcarrier activation patterns (SAPs) implicitly convey additional information bits that are known as IM bits. By deactivating partial subcarriers, some transmit power can be saved, which can be used in return for the transmission of $M$-ary modulation symbols. The transmission of IM bits consumes no power and has stronger protection than that of ordinary modulation bits [22]. Therefore, OFDM-IM achieves better error performance than its OFDM counterpart. Moreover, OFDM-IM enjoys the flexibility in system configuration and provides a good trade-off between the spectral efficiency and the system performance by adjusting the number of active subcarriers. To extend the wireless coverage, relay-aided OFDM-IM is proposed. In [23], adaptive mapping schemes between IM bits and SAPs are presented for dual-hop OFDMIM DF relaying networks. To enhance the system reliability, in [24]-[26], multiple relays with relay selection are employed for dual-hop OFDM-IM. In contrast to the dual-hop schemes in [23]-[26], multi-hop DF relaying is developed for OFDMIM in [27], further enlarging the communication coverage. In view of two information-carrying units, the potential of OFDM-IM is investigated in overlay OFDM-based CR networks, where the PU transmits classical OFDM signals, and the SU employs OFDM-IM to forward the PU's information via $M$-ary symbols and convey its own information through SAPs [28]. However, how to explore the opportunity to access the idle spectrum when the PU employs OFDM-IM for information transmission, is still an open topic. In particular, since partial subcarriers in OFDM-IM are inherently inactive (spectrum holes), they can potentially be used by SUs for improving the spectral efficiency.

Against the background, in this paper, we propose a novel OFDM-IM-based CR (OFDM-IM-CR) model for spectrum sharing to improve spectrum utilization. The contributions of this paper are summarized as follows.

- In OFDM-IM-CR, the PU relies on dual-hop OFDM-IM AF (OFDM-IM-AF) relaying for priority transmission and the SU exploits spectrum holes of the primary network for opportunistic communications. Specifically, in the first phase, the primary transmitter (PT) broadcasts OFDM-IM signals to an AF relay and the secondary transmitter (ST). Upon receiving this signal, the ST measures the signal-to-noise ratio (SNR) and performs spectrum sensing. In the second phase, the relay amplifies and forwards the received signal to the primary receiver (PR). Meanwhile, if the SNR of the PT $\rightarrow \mathrm{ST}$ link is above a predefined threshold, the ST transmits its own information on those inactive subcarriers of the primary network to the secondary receiver (SR); otherwise, the
ST stays in silent mode.

- Two different types of maximum-likelihood (ML) detectors are designed for the primary network, based on the knowledge of either the estimated channel state information (CSI) or the statistical channel information of the secondary network. A complexity-reducing method applicable to both types is proposed, and is shown to exhibit near optimal performance. Bit error rate (BER) analysis is provided for the PU and a tight upper bound on the BER is derived, assuming the first type of ML detection.

- The accuracy of our analysis and the effectiveness of OFDM-IM-CR are verified through Monte Carlo simulations, whose results show that OFDM-IM-CR has the potential of outperforming OFDM-CR and OFDM-IM$\mathrm{AF}$ in terms of BER with higher spectral efficiency. The two proposed types of detectors provide different tradeoffs between the error performance, detection complexity, and requirement of CSI. In the presence of channel estimation errors of the secondary network, the first type performs better at low SNR, while the second type is preferable at high SNR.

The remainder of this paper is organized as follows. The system model of OFDM-IM-CR is described in Section II. Section III presents two types of ML detectors, followed by the performance analysis in Section IV. Section V gives computer simulation results and finally Section VI concludes the paper.

Notation: Column vectors are in the form of lowercase bold letters. $\mathbf{I}_{n}$ denotes the $n \times n$ identity matrix. Superscript ${ }^{T}$ stands for transpose. $\Re\{\cdot\}$ returns the real part of a complex number. $\mathcal{C N}\left(0, \sigma^{2}\right)$ represents the complex Gaussian distribution with zero mean and variance $\sigma^{2}$. The probability of an event and the probability distribution function (PDF) of a random variable are denoted by $\operatorname{Pr}(\cdot)$ and $f(\cdot)$, respectively. $E\{\cdot\}$ denotes expectation. $\lfloor\cdot\rfloor$ and $C(\cdot, \cdot)$ are the floor function and the binomial coefficient, respectively. $Q(\cdot)$ and $\operatorname{Ei}(\cdot)$ represent the Gaussian $Q$-function and the exponential integral function, respectively.

\section{SYSTEM MODEL}

In this section, we present the system model of OFDM-IM$\mathrm{CR}$, including single-PU/SU and multi-PU/SU scenarios.

\section{A. Single-PU/SU Scenario}

Fig. 1 depicts the system model of OFDM-IM-CR in the single-PU/SU scenario, where the ST is close to the PT, and all nodes operate in the half-duplex mode with a single transmit/receive antenna. Due to the broadband signal and rich scattering in the propagation environment, all channel links are assumed to experience frequency-selective Rayleigh fading, except the PT $\rightarrow \mathrm{ST}$ link, which is modeled by frequencyselective Rician fading as the ST and PT are located close together.

The complete communication protocol comprises two phases. In the first phase, the PT transmits its information via OFDM-IM signals to the relay $(\mathrm{R})$, which is also overheard by the ST. According to [16], the formation of the OFDM-IM 


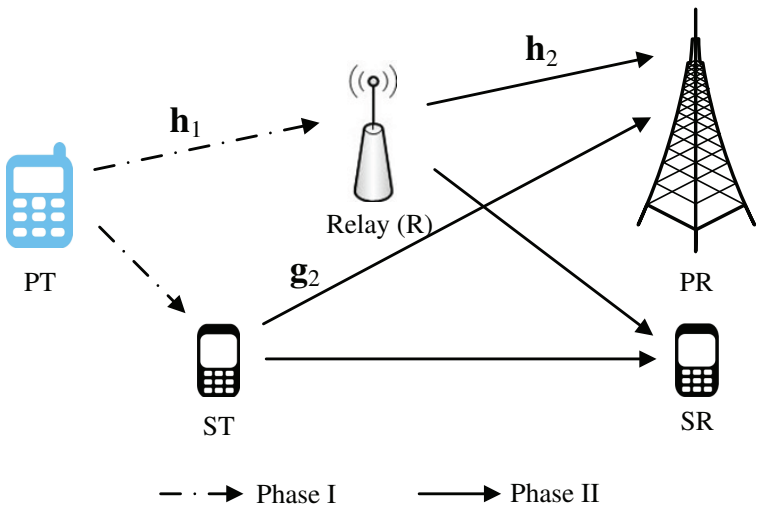

Fig. 1. System model of OFDM-IM-CR.

signal is the same as that of OFDM except that IM is included in the generation of the frequency-domain OFDM block. Specifically, for an OFDM-IM system with $N$ subcarriers, the $N \times 1$ main OFDM block is created by stacking $G$ subblocks, each of which is formed by performing IM within $n=N / G$ subcarriers. Since the processes for all subblocks are the same and independent of each other, we focus only on a single subblock, say, the $\beta$-th one, where $\beta \in\{1, \ldots, G\}$. The information bits to be transmitted in the $\beta$-th subblock are divided into two parts for further processing. The first part, consisting of $p_{1}=\left\lfloor\log _{2}(C(n, k))\right\rfloor$ bits, is used to determine the positions of $k$ active subcarriers within the $\beta$-th subblock, forming the active indices (i.e., the SAP)

$$
\mathcal{I}^{(\beta)}=\left\{i^{(\beta)}(1), \ldots, i^{(\beta)}(k)\right\},
$$

where $i^{(\beta)}(\lambda) \in\{1, \ldots, n\}$ with $\lambda=1, \ldots, k$. In other words, $\mathcal{I}^{(\beta)}$ has $c=2^{p_{1}}$ possible realizations. The mapping between the $p_{1}$ bits and $\mathcal{I}^{(\beta)}$ can be easily realized by either a look-up table or the combinatorial method [16]. The second part with $p_{2}=k \log _{2}(M)$ bits is mapped to the data symbol vector

$$
\mathbf{s}_{p}^{(\beta)}=\left[s_{p}^{(\beta)}(1), \ldots, s_{p}^{(\beta)}(k)\right]^{T},
$$

according to a normalized $M$-ary phase shift keying ( $\mathrm{P}$ $\mathrm{SK})$ /quadrature amplitude modulation (QAM) signal constellation $\mathcal{X}$, where $s_{p}^{(\beta)}(\lambda) \in \mathcal{X}$ is to be transmitted over the subcarrier with index $i^{(\beta)}(\lambda)$. After obtaining $\mathcal{I}^{(\beta)}$ and $\mathbf{s}^{(\beta)}$ for all $\beta$, we have the $N \times 1$ main OFDM block for the PT as

$$
\mathbf{x}_{p t}=\left[x_{p}(1), \ldots, x_{p}(N)\right]^{T}=\left[\left(\mathbf{x}_{p}^{(1)}\right)^{T}, \ldots,\left(\mathbf{x}_{p}^{(G)}\right)^{T}\right]^{T},
$$

where $\mathbf{x}_{p}^{(\beta)}=\left[x_{p}^{(\beta)}(1), \ldots, x_{p}^{(\beta)}(n)\right]^{T}$ is the $\beta$-th $n \times 1$ subblock with $x_{p}^{(\beta)}(\alpha) \in\{0, \mathcal{X}\}, \alpha=1, \ldots, n$. After this point, the remaining procedures, which mainly include the $N$ point inverse discrete Fourier transform (DFT), cyclic prefix (CP) appending, and parallel-to-serial conversion, are carried out in sequence and then the data is broadcasted to $\mathrm{R}$ and the ST.

At the ST, the received SNR is measured and spectrum sensing is performed to detect the SAP. At R, after discarding the $\mathrm{CP}$ and performing $N$-point DFT, the received signal for the $\beta$-th subblock in the frequency domain can be expressed as

$$
y_{1}^{\beta}(\alpha)=h_{1}^{(\beta)}(\alpha) x_{p}^{(\beta)}(\alpha)+w_{1}^{(\beta)}(\alpha), \quad \alpha=1, \ldots, n,
$$

where $h_{1}^{(\beta)}(\alpha)$ and $w_{1}^{(\beta)}(\alpha)$, respectively, are the channel frequency response (CFR) of the $\mathrm{PT} \rightarrow \mathrm{R}$ link and the additive white Gaussian noise (AWGN) sample at $\mathrm{R}$ on the $\alpha$-th subcarrier of the $\beta$-th subblock. It is assumed that $h_{1}^{(\beta)}(\alpha)$ and $w_{1}^{(\beta)}(\alpha)$ follow the distributions $\mathcal{C N}\left(0, \sigma_{1}^{2}\right)$ and $\mathcal{C N}\left(0, N_{0}\right)$, respectively. We define the transmit SNR per subcarrier as $\rho=k /\left(n N_{0}\right)$.

In the second phase, $\mathrm{R}$ forwards $y_{1}^{(\beta)}(\alpha), \beta=1, \ldots, G, \alpha=$ $1, \ldots, n$, to the PR and SR with an amplification factor $A$. With the fixed-gain AF relaying strategy [29], $A$ can be written as

$$
A=\sqrt{\frac{P_{R}}{P_{S} \sigma_{1}^{2}+N_{0}}},
$$

where $P_{S}=k / n$ is the transmit power per subcarrier of the $\mathrm{PT}$, and $P_{R}$ denotes the transmit power per subcarrier of the relay. Meanwhile, if the value of the received SNR at the ST of the first phase is above a predefined threshold, it is reasonable to assume that perfect spectrum sensing can be achieved and the ST correctly obtains the positions of the inactive subcarriers of the PU, i.e., $\overline{\mathcal{I}}^{(\beta)}=\left\{\bar{i}^{(\beta)}(1), \ldots, \bar{i}^{(\beta)}(n-\right.$ $k)\}=\{1, \ldots, n\} \backslash \mathcal{I}^{(\beta)}$, where $\bar{i}^{(\beta)}(\eta) \in\{1, \ldots, n\}$ with $\eta=1, \ldots, n-k$. After that, the ST modulates its own information of $p_{3}=(n-k) \log _{2}(M)$ bits into the symbol vector $^{1}$

$$
\mathbf{s}_{s}^{(\beta)}=\left[s_{s}^{(\beta)}(1), \ldots, s_{s}^{(\beta)}(n-k)\right]^{T},
$$

where $s_{s}^{(\beta)}(\eta) \in \mathcal{X}$ is to be transmitted over the subcarrier of index $\bar{i}^{(\beta)}(\eta)$. Concatenating all $G$ subblocks yields the $N \times 1$ main OFDM block for the ST, namely

$$
\mathbf{x}_{s t}=\left[x_{s}(1), \ldots, x_{s}(N)\right]^{T}=\left[\left(\mathbf{x}_{s}^{(1)}\right)^{T}, \ldots,\left(\mathbf{x}_{s}^{(G)}\right)^{T}\right]^{T},
$$

and then $\mathbf{x}_{s t}$ is broadcasted to the SR and PR, where $\mathbf{x}_{s}^{(\beta)}=$ $\left[x_{s}^{(\beta)}(1), \ldots, x_{s}^{(\beta)}(n)\right]^{T}$ is the $\beta$-th $n \times 1$ subblock with $x_{s}^{(\beta)}(\alpha) \in\{0, \mathcal{X}\}, \alpha=1, \ldots, n$. Note that the indices of nonzero elements in $\mathbf{x}_{s t}$ are complementary to those in $\mathbf{x}_{p t}$.

The signals from $\mathrm{R}$ and the ST arrive at the PR simultaneously, such that the received signal for the $\beta$-th subblock in the frequency domain is given by

$$
\begin{aligned}
y_{2}^{(\beta)}(\alpha)= & A h_{2}^{(\beta)}(\alpha) y_{1}^{(\beta)}(\alpha)+g_{2}^{(\beta)}(\alpha) x_{s}^{(\beta)}(\alpha)+w_{2}^{(\beta)}(\alpha) \\
= & A h_{1}^{(\beta)}(\alpha) h_{2}^{(\beta)}(\alpha) x_{p}^{(\beta)}(\alpha)+g_{2}^{(\beta)}(\alpha) x_{s}^{(\beta)}(\alpha) \\
& +A h_{2}^{(\beta)}(\alpha) w_{1}^{(\beta)}(\alpha)+w_{2}^{(\beta)}(\alpha), \quad \alpha=1, \ldots, n,
\end{aligned}
$$

where $h_{2}^{(\beta)}(\alpha)$ and $g_{2}^{(\beta)}(\alpha)$, respectively, are the CFRs of the $\mathrm{R} \rightarrow \mathrm{PR}$ and $\mathrm{ST} \rightarrow \mathrm{PR}$ links on the $\alpha$-th subcarrier of

\footnotetext{
${ }^{1}$ The ST stays in silent mode if the value of the received SNR of the first phase is below the threshold. In this case, the SU fails to access the spectrum of the PU, and the proposed OFDM-IM-CR reduces to OFDM-IM-AF.
} 
the $\beta$-th subblock, following the distributions $\mathcal{C N}\left(0, \sigma_{2}^{2}\right)$ and $\mathcal{C N}\left(0, \phi_{2}^{2}\right) ; w_{2}^{(\beta)}(\alpha)$ is the AWGN sample at the PR on the $\alpha$-th subcarrier of the $\beta$-th subblock with the distribution $\mathcal{C N}\left(0, N_{0}\right)$. After noise normalization, (8) can be rewritten as

$$
\begin{aligned}
\bar{y}_{2}^{(\beta)}(\alpha)= & A h_{1}^{(\beta)}(\alpha) h_{2}^{(\beta)}(\alpha) r^{(\beta)}(\alpha) x_{p}^{(\beta)}(\alpha) \\
& +g_{2}^{(\beta)}(\alpha) r^{(\beta)}(\alpha) x_{s}^{(\beta)}(\alpha)+w^{(\beta)}(\alpha),
\end{aligned}
$$

where $r^{(\beta)}(\alpha)=1 / \sqrt{1+A^{2}\left|h_{2}^{(\beta)}(\alpha)\right|^{2}}$ and $w^{(\beta)}(\alpha)$ is a complex-valued Gaussian variable with zero mean and variance $N_{0}$.

Similar to (9), the received signal at the SR can be easily formulated, which is omitted here since we mainly study the impact of the secondary network on the primary one in this paper.

\section{B. Multi-PU/SU Scenario}

In this subsection, we extend the single-PU/SU scenario to a multi-PU/SU scenario by resorting to the idea of OFDM access.

For the multi-PU/SU scenario, the total frequency band is partitioned into $N$ orthogonal subcarriers, and every $n=N / G$ adjacent subcarriers compose a subcarrier group, where $G$ is the number of subcarrier groups. In particular, each subcarrier group is then exclusively dedicated to serving one PU and one SU, and each PU and its corresponding SU constitute a basic unit of the network. As a result, $G$ PUs and $G$ SUs can be supported by the network. Since the $G$ units operate over orthogonal subcarrier groups, no co-channel interference occurs between any two units. Obviously, in each unit, the PU and the SU function the same as those in the single-PU/SU scenario, i.e., the PU relies on OFDM-IM-AF relaying for priority transmission and the SU exploits the spectrum holes of the primary network for opportunistic communications. The signal model for the $g$-th unit is the same as that for the $g$-th subblock in the single-PU/SU scenario, where $g \in\{1, \ldots, G\}$. Therefore, the receiver design, performance analysis, and computer simulations for the multi-user scenario are straightforward, which are omitted in this paper to avoid redundancy.

Remark 1: The ML detector [16] and the energy detector [30] can be used for spectrum sensing (SAP detection) at the ST. The ML detector makes a joint decision on the SAP and the constellation symbols by searching all possible combinations of them. It is the optimal detector, and achieves a diversity order of two for the SAP detection. However, this detector necessitates the CSI of the PT $\rightarrow$ ST link at the ST, and leads to high computational complexity. The energy detector measures the received energy on each subcarrier, and then selects $k$ subcarriers having the largest energies as the active subcarriers. For the energy detector, the CSI is no longer required at the $\mathrm{ST}$, and the computational complexity is much lower. The drawback is that it suffers from a loss of diversity gain and performs much worse than the ML detector.

Remark 2: Though not shown in this paper, practical spectrum sensing methods, such as the ML and energy detectors, are able to approach and even achieve the optimal overall performance that is obtained with perfect spectrum sensing over Rician fading channels. For this reason and for ease of performance analysis for perfect spectrum sensing, we assume perfect spectrum sensing instead of practical spectrum sensing at the ST in this paper.

\section{RECEIVER DESIGN}

We observe that the primary network with the ST can be regarded as an uplink multi-user communication system. Multi-user or single-user detector can be employed at the PR, depending on the amount of CSI. In this section, provided that the PR has partial CSI of the secondary network as well as perfect CSI of its own network ${ }^{2}$, we design two types of ML detectors for the PR, which utilize the estimated CSI and statistical channel information of the $\mathrm{ST} \rightarrow \mathrm{PR}$ link, respectively. They provide different trade-offs between the error performance, detection complexity, and requirement of CSI. Moreover, a complexity-reducing method applicable to both types is also proposed, and this method exhibits near optimal performance. Due to the encoding independence among different subblocks, the detection for all subblocks can be performed in parallel, and the superscript $(\beta)$ is omitted for brevity in the sequel. We define $\mathbf{h}_{1}=\left[h_{1}(1), \ldots, h_{1}(n)\right]^{T}$, $\mathbf{h}_{2}=\left[h_{2}(1), \ldots, h_{2}(n)\right]^{T}$, and $\mathbf{g}_{2}=\left[g_{2}(1), \ldots, g_{2}(n)\right]^{T}$.

\section{A. ML Detector with Estimated CSI of $\mathbf{g}_{2}$}

In this subsection, assuming that perfect knowledge of the CSI of $\mathbf{h}_{1}$ and $\mathbf{h}_{2}$ is available at the PR, we deal with the detection problem of the PU in the presence of channel estimation errors of $\mathbf{g}_{2}$.

From (9), the mismatched ML detector at the PR with channel estimation errors of $\mathbf{g}_{2}$ makes a joint decision on $\mathcal{I}$, $\mathbf{s}_{p}$, and $\mathbf{s}_{s}$ by searching all possible combinations of them, namely

$$
\begin{aligned}
\left(\hat{\mathcal{I}}, \hat{\mathbf{s}}_{p}, \hat{\mathbf{s}}_{s}\right)=\underset{\mathcal{I}, \mathbf{s}_{p}, \mathbf{s}_{s}}{\arg \min } \sum_{\alpha=1}^{n} \mid & \bar{y}_{2}(\alpha) \\
& -A h_{1}(\alpha) h_{2}(\alpha) r(\alpha) x_{p}(\alpha) \\
& -\left.\hat{g}_{2}(\alpha) r(\alpha) x_{s}(\alpha)\right|^{2}
\end{aligned}
$$

where $\hat{\mathcal{I}}=\{\hat{i}(1), \ldots, \hat{i}(k)\}, \hat{\mathbf{s}}_{p}=\left[\hat{s}_{p}(1), \ldots, \hat{s}_{p}(k)\right]^{T}$, and $\hat{\mathbf{s}}_{s}=\left[\hat{s}_{s}(1), \ldots, \hat{s}_{s}(n-k)\right]^{T}$ are the estimates of $\mathcal{I}, \mathbf{s}_{p}$, and $\mathbf{s}_{s}$, respectively. In (10), $\hat{\mathbf{g}}_{2}$ is the vector of estimated channel coefficients, which is given by

$$
\hat{\mathbf{g}}_{2}=\left[\hat{g}_{2}(1), \ldots, \hat{g}_{2}(n)\right]^{T}=\mathbf{g}_{2}+\mathbf{g}_{e},
$$

where $\mathbf{g}_{e}=\left[g_{e}(1), \ldots, g_{e}(n)\right]^{T}$ denotes the $n \times 1$ vector of channel estimation errors with the distribution $\mathcal{C N}\left(\mathbf{0}, \phi_{e}^{2} \mathbf{I}_{n}\right)$, and it is independent of $\mathbf{g}_{2}$. Hence, $\hat{\mathbf{g}}_{2}$ follows the distribution $\mathcal{C N}\left(\mathbf{0},\left(\phi_{2}^{2}+\phi_{e}^{2}\right) \mathbf{I}_{n}\right)$.

\footnotetext{
${ }^{2}$ Since OFDM-IM-CR is an underlay CR paradigm, the power of the PT is often larger than that of the ST. Moreover, the primary links are always included in the network, while the presence of the secondary network is opportunistic. Hence, the channel estimation of the primary network can be more reliable than that of the secondary network at the PR
} 
After careful observation, we find that the objective function in (10) can be further rewritten as

$$
\begin{aligned}
& \min _{\mathcal{I}, \mathbf{s}_{p}, \mathbf{s}_{s}}( \sum_{\alpha \in \mathcal{I}}\left|\bar{y}_{2}(\alpha)-A h_{1}(\alpha) h_{2}(\alpha) r(\alpha) x_{p}(\alpha)\right|^{2} \\
&\left.+\sum_{\alpha \in \overline{\mathcal{I}}}\left|\bar{y}_{2}(\alpha)-\hat{g}_{2}(\alpha) r(\alpha) x_{s}(\alpha)\right|^{2}\right) \\
&=\min _{\mathcal{I}}\left(\sum_{\alpha \in \mathcal{I}} \min _{u(\alpha) \in \mathcal{X}}\left|\bar{y}_{2}(\alpha)-A h_{1}(\alpha) h_{2}(\alpha) r(\alpha) u(\alpha)\right|^{2}\right. \\
&\left.+\sum_{\alpha \in \overline{\mathcal{I}}} \min _{v(\alpha) \in \mathcal{X}}\left|\bar{y}_{2}(\alpha)-\hat{g}_{2}(\alpha) r(\alpha) v(\alpha)\right|^{2}\right) \\
&=\min _{\mathcal{I}}\left(\sum_{\alpha \in \mathcal{I}} D_{1}(\alpha)+\sum_{\alpha \in \overline{\mathcal{I}}} D_{2}(\alpha)\right)
\end{aligned}
$$

where

$$
D_{1}(\alpha)=\left|\bar{y}_{2}(\alpha)-A h_{1}(\alpha) h_{2}(\alpha) r(\alpha) \hat{u}(\alpha)\right|^{2}
$$

and

$$
D_{2}(\alpha)=\left|\bar{y}_{2}(\alpha)-\hat{g}_{2}(\alpha) r(\alpha) \hat{v}(\alpha)\right|^{2}
$$

with

$$
\hat{u}(\alpha)=\underset{u(\alpha) \in \mathcal{X}}{\arg \min }\left|\bar{y}_{2}(\alpha)-A h_{1}(\alpha) h_{2}(\alpha) r(\alpha) u(\alpha)\right|^{2},
$$

and

$$
\hat{v}(\alpha)=\underset{v(\alpha) \in \mathcal{X}}{\arg \min }\left|\bar{y}_{2}(\alpha)-\hat{g}_{2}(\alpha) r(\alpha) v(\alpha)\right|^{2} .
$$

Therefore, we have

$$
\hat{\mathcal{I}}=\underset{\mathcal{I}}{\arg \min }\left(\sum_{\alpha \in \mathcal{I}} D_{1}(\alpha)+\sum_{\alpha \in \overline{\mathcal{I}}} D_{2}(\alpha)\right),
$$

and

$$
\hat{s}_{p}(\lambda)=\hat{u}(\hat{i}(\lambda)), \quad \lambda=1, \ldots, k
$$

It is easy to observe from (12)-(18) that the overall computational complexity of the ML detector is of order $\sim \mathcal{O}(c+$ $2 n M)$ per subblock in terms of complex multiplications.

\section{B. ML Detector with Statistical Channel Information of $\mathbf{g}_{2}$}

In this subsection, we consider the scenario in which the PR has the perfect knowledge of $\mathbf{h}_{1}$ and $\mathbf{h}_{2}$, and statistical information about $\mathbf{g}_{2}$. According to (9), the PDF of $\bar{y}_{2}(\alpha)$ is given by (19), shown at the top of the next page, where $N_{0}^{\prime}(\alpha)=N_{0}+\phi_{2}^{2} r^{2}(\alpha)$. Therefore, from (19), the ML detector can be formulated as

$$
\begin{aligned}
\left(\hat{\mathcal{I}}, \hat{\mathbf{s}}_{p}\right)=\underset{\mathcal{I}, \mathbf{s}_{p}}{\arg \min }( & \frac{1}{N_{0}} \sum_{\alpha \in \mathcal{I}} \mid \bar{y}_{2}(\alpha) \\
& -\left.A h_{1}(\alpha) h_{2}(\alpha) r(\alpha) x_{p}(\alpha)\right|^{2} \\
& \left.+\sum_{\alpha \in \overline{\mathcal{I}}} \frac{\left|\bar{y}_{2}(\alpha)\right|^{2}}{N_{0}^{\prime}(\alpha)}+\ln N_{0}^{\prime}(\alpha)\right) \\
= & \underset{\mathcal{I}}{\arg \min }\left(\sum_{\alpha \in \mathcal{I}} D_{1}^{\prime}(\alpha)+\sum_{\alpha \in \overline{\mathcal{I}}} D_{2}^{\prime}(\alpha)\right)
\end{aligned}
$$

where $D_{1}^{\prime}(\alpha)=D_{1}(\alpha) / N_{0}, D_{2}^{\prime}(\alpha)=\left|\bar{y}_{2}(\alpha)\right|^{2} / N_{0}^{\prime}(\alpha)+$ $\ln N_{0}^{\prime}(\alpha)$, and $\hat{\mathbf{s}}_{p}$ is given by (18). Obviously, the overall computational complexity of this detector is of order $\sim \mathcal{O}(c+$ $n M)$ per subblock in terms of complex multiplications.

\section{Low-Complexity Detectors}

As shown in Sections III.A and III.B, the detection of $\mathcal{I}$ in both types of ML detectors is based on the exhaustive search over all possible realizations of $\mathcal{I}$, which is impractical for large values of $c$. To this end, we develop a complexityreducing method for SAP detection, which is applicable to both types and achieves near optimal performance. Without loss of generality, we take the first type as an illustrative example in this subsection.

First, let us take a deeper look at (17). The difference of two outputs of the objection function in (17) with the inputs of any two different SAPs, denoted by $\mathcal{I}$ and $\mathcal{I}^{\prime}$, is given by

$$
\begin{aligned}
& {\left[\sum_{\alpha \in \mathcal{I}} D_{1}(\alpha)+\sum_{\alpha \in \overline{\mathcal{I}}} D_{2}(\alpha)\right]-\left[\sum_{\alpha \in \mathcal{I}^{\prime}} D_{1}(\alpha)+\sum_{\alpha \in \overline{\mathcal{I}}^{\prime}} D_{2}(\alpha)\right]} \\
& =\sum_{\alpha \in \mathcal{I} \backslash\left(\mathcal{I} \cap \mathcal{I}^{\prime}\right)} D_{1}(\alpha)+\sum_{\alpha \in \overline{\mathcal{I}} \backslash\left(\overline{\mathcal{I}} \cap \overline{\mathcal{I}}^{\prime}\right)} D_{2}(\alpha) \\
& \quad-\sum_{\alpha \in \mathcal{I}^{\prime} \backslash\left(\mathcal{I} \cap \mathcal{I}^{\prime}\right)} D_{1}(\alpha)-\sum_{\alpha \in \overline{\mathcal{I}}^{\prime} \backslash\left(\overline{\mathcal{I}} \cap \overline{\mathcal{I}}^{\prime}\right)} D_{2}(\alpha) \\
& =\left[\sum_{\alpha \in \mathcal{I} \backslash\left(\mathcal{I} \cap \mathcal{I}^{\prime}\right)} D_{1}(\alpha)-D_{2}(\alpha)\right]
\end{aligned}
$$$$
-\left[\sum_{\alpha \in \mathcal{I}^{\prime} \backslash\left(\mathcal{I} \cap \mathcal{I}^{\prime}\right)} D_{1}(\alpha)-D_{2}(\alpha)\right]
$$$$
=\left[\sum_{\alpha \in \mathcal{I}} D_{1}(\alpha)-D_{2}(\alpha)\right]-\left[\sum_{\alpha \in \mathcal{I}^{\prime}} D_{1}(\alpha)-D_{2}(\alpha)\right] \text {. }
$$

From (21), we conclude that (17) is equivalent to

$$
\hat{\mathcal{I}}=\underset{\mathcal{I}}{\arg \min } \sum_{\alpha \in \mathcal{I}} D_{1}(\alpha)-D_{2}(\alpha) .
$$

Based on (22), we propose a low-complexity near-ML detector, which simply selects $k$ indices out of $n$ that have minimum values of $D_{1}(\alpha)-D_{2}(\alpha)$ as $\hat{\mathcal{I}}$, and obtains the estimate of $\mathbf{s}_{p}$ from (18). Although an illegal $\hat{\mathcal{I}}$ may be attained by this simplified near-ML detector due to the presence of 


$$
f\left(\bar{y}_{2}(\alpha)\right)= \begin{cases}\frac{1}{\pi N_{0}} \exp \left(-\frac{1}{N_{0}}\left|\bar{y}_{2}(\alpha)-A h_{1}(\alpha) h_{2}(\alpha) r(\alpha) x_{p}(\alpha)\right|^{2}\right), & \text { for } \alpha \in \mathcal{I} \\ \frac{1}{\pi N_{0}^{\prime}(\alpha)} \exp \left(-\frac{1}{N_{0}^{\prime}(\alpha)}\left|\bar{y}_{2}(\alpha)\right|^{2}\right), & \text { for } \alpha \in \overline{\mathcal{I}}\end{cases}
$$

$C(n, k)-c$ unused SAPs at the transmitter, the probability of this event is quite small, and as will be shown in Section V, the resulting performance loss is negligible. More importantly, the computational complexity is substantially reduced to $\sim$ $\mathcal{O}(2 n M)$ per subblock, in terms of complex multiplications.

We would like to note that the above-mentioned near-ML detector can also be derived from a simplified log-likelihood ratio (LLR) detector, as described below. Since each subcarrier carries the symbol from either the PT or ST, the exact LLR is given by

$$
L(\alpha)=\ln \frac{\operatorname{Pr}\left(E \mid \bar{y}_{2}(\alpha)\right)}{\operatorname{Pr}\left(\bar{E} \mid \bar{y}_{2}(\alpha)\right)}=\ln \frac{\operatorname{Pr}(E) f\left(\bar{y}_{2}(\alpha) \mid E\right)}{\operatorname{Pr}(\bar{E}) f\left(\bar{y}_{2}(\alpha) \mid \bar{E}\right)}
$$

for $\alpha=1, \ldots, n$, where $E$ denotes the event that the $\alpha$-th subcarrier conveys the symbol from the PT, and $\bar{E}$ is the complement of $E$. From (23), a larger value of $L(\alpha)$ means that the $\alpha$-th subcarrier is more likely to convey the symbol from the PT. Since $k$ out of $n$ subcarriers carry symbols from the PT and $n-k$ from the ST, it follows that $\operatorname{Pr}(E)=k / n$ and $\operatorname{Pr}(\bar{E})=(n-k) / n$. Hence, (23) reduces to (24), shown at the top of the next page, where $\mathcal{C}=\ln (k)-\ln (n-k)$ is a constant irrelevant to the detection. By resorting to (13)-(16), $L(\alpha)$ in (24) can be well approximated as

$$
\begin{aligned}
L(\alpha) \approx & \frac{1}{N_{0}}\left|\bar{y}_{2}(\alpha)-\hat{g}_{2}(\alpha) r(\alpha) \hat{v}(\alpha)\right|^{2} \\
& -\frac{1}{N_{0}}\left|\bar{y}_{2}(\alpha)-A h_{1}(\alpha) h_{2}(\alpha) r(\alpha) \hat{u}(\alpha)\right|^{2}+\mathcal{C} \\
\propto & D_{2}(\alpha)-D_{1}(\alpha) .
\end{aligned}
$$

Hence, the calculation of the LLR is significantly simplified as the subtraction of two minimum Euclidean distances. After obtaining the $n$ LLR values via (25), the estimate of $\mathcal{I}$ can be easily derived by choosing $k$ indices having maximum LLR values, which is in fact identical to the near-ML detector.

We note that the first and second types of detectors can be regarded as multi-user and single-user detectors, respectively. Since the error probability of the single-user detector is extremely difficult to evaluate as in conventional multi-user communications, in the next section, we will concentrate on the performance analysis of the ML detector designed in (10).

\section{ERror PERFORMANCE ANALYSis}

In this section, we derive an upper bound on the BER of the PU that utilizes the joint ML detector designed in (10) by taking into account the channel estimation errors of $\mathbf{g}_{2}$.

Obviously, the bit errors of the PU can be classified into two cases: (i) an incorrect estimate of the SAP, and (ii) a correct estimate of the SAP. Therefore, the bit error probability (BEP) of the PU can be written in the form of

$$
P_{u} \approx P_{I} \frac{\zeta p_{1}+\frac{1}{2} p_{2}}{p_{1}+p_{2}}+\left(1-P_{I}\right) \frac{p_{2} P_{s b}}{p_{1}+p_{2}}
$$

where $P_{I}$ refers to the error probability of the SAP detection; $\zeta=1$ for $n=2$, while $\zeta=1 / 2$ for $n>2 ; P_{s b}$ denotes the BEP of $M$-ary PSK/QAM demodulation in dualhop AF relaying over Rayleigh fading channels. In (26), the approximation results from the first term on the right-hand side, which describes the BEP for the case where the SAP is detected erroneously. In this case, errors occur in both the IM bits and the constellation bits. For the IM bits, when $(n, k)=(2,1)$, there are only two possible SAPs $\left(p_{1}=1\right)$ such that we have $\zeta=1$, namely the IM bit is certainly wrong; when $n>2$, since the mapping between the IM bits and SAPs does not provide Gray codes, half of the IM bits can be approximated incorrectly [31]. For the constellation bits, since the BEP is dominated by that of $M$-ary symbols whose associated subcarriers are incorrectly estimated as inactive, and the detector tries to recover those $M$-ary symbols from pure noise, the BEP can thereby be approximated as $1 / 2$. The calculation of $P_{I}$ and $P_{s b}$ will be addressed in the next two subsections, respectively.

\section{A. Calculation of $P_{I}$}

Let us first consider the conditional pairwise error probability (PEP), i.e., $\operatorname{Pr}\left(\mathbf{x}_{p} \rightarrow \hat{\mathbf{x}}_{p}, \mathbf{x}_{s} \rightarrow \hat{\mathbf{x}}_{s} \mid \mathbf{h}_{1}, \mathbf{h}_{2}, \hat{\mathbf{g}}_{2}\right)$, which represents the probability of the event that the transmitted $\mathbf{x}_{p}$ and $\mathbf{x}_{s}$ are erroneously detected as $\hat{\mathbf{x}}_{p}$ and $\hat{\mathbf{x}}_{s}$, respectively, conditioned on $\mathbf{h}_{1}, \mathbf{h}_{2}$, and $\mathbf{g}_{2}$. From (10), we have

$$
\begin{gathered}
\operatorname{Pr}\left(\mathbf{x}_{p} \rightarrow \hat{\mathbf{x}}_{p}, \mathbf{x}_{s} \rightarrow \hat{\mathbf{x}}_{s} \mid \mathbf{h}_{1}, \mathbf{h}_{2}, \hat{\mathbf{g}}_{2}\right) \\
=\operatorname{Pr}\left(\sum_{\alpha=1}^{n} \mid \bar{y}_{2}(\alpha)-A h_{1}(\alpha) h_{2}(\alpha) r(\alpha) x_{p}(\alpha)\right. \\
\quad-\left.\hat{g}_{2}(\alpha) r(\alpha) x_{s}(\alpha)\right|^{2} \\
>\sum_{\alpha=1}^{n} \mid \bar{y}_{2}(\alpha)-A h_{1}(\alpha) h_{2}(\alpha) r(\alpha) \hat{x}_{p}(\alpha) \\
\left.\quad-\left.\hat{g}_{2}(\alpha) r(\alpha) \hat{x}_{s}(\alpha)\right|^{2}\right) \\
=\operatorname{Pr}\left(\sum_{\alpha=1}^{n}|x(\alpha)|^{2}-2 \Re\left\{\bar{y}_{2}^{*}(\alpha) x(\alpha)\right\}\right. \\
\left.>\sum_{\alpha=1}^{n}|\hat{x}(\alpha)|^{2}-2 \Re\left\{\bar{y}_{2}^{*}(\alpha) \hat{x}(\alpha)\right\}\right),
\end{gathered}
$$

where

$$
x(\alpha)=A h_{1}(\alpha) h_{2}(\alpha) r(\alpha) x_{p}(\alpha)+\hat{g}_{2}(\alpha) r(\alpha) x_{s}(\alpha)
$$

and

$$
\hat{x}(\alpha)=A h_{1}(\alpha) h_{2}(\alpha) r(\alpha) \hat{x}_{p}(\alpha)+\hat{g}_{2}(\alpha) r(\alpha) \hat{x}_{s}(\alpha) .
$$




$$
L(\alpha)=\ln \left(\sum_{\iota=1}^{M} \exp \left(-\frac{1}{N_{0}}\left|\bar{y}_{2}(\alpha)-A h_{1}(\alpha) h_{2}(\alpha) r(\alpha) s_{\iota}\right|^{2}\right)\right)-\ln \left(\sum_{\iota=1}^{M} \exp \left(-\frac{1}{N_{0}}\left|\bar{y}_{2}(\alpha)-\hat{g}_{2}(\alpha) r(\alpha) s_{\iota}\right|^{2}\right)\right)+\mathcal{C},
$$

With (11), (9) can be rewritten as

$$
\begin{aligned}
\bar{y}_{2}(\alpha)= & A h_{1}(\alpha) h_{2}(\alpha) r(\alpha) x_{p}(\alpha)+\hat{g}_{2}(\alpha) r(\alpha) x_{s}(\alpha) \\
& -g_{e}(\alpha) r(\alpha) x_{s}(\alpha)+w(\alpha) \\
= & x(\alpha)+\bar{w}(\alpha),
\end{aligned}
$$

where

$$
\bar{w}(\alpha)=-g_{e}(\alpha) r(\alpha) x_{s}(\alpha)+w(\alpha) .
$$

Substituting (30) into (27) yields (32), which is shown at the top of the next page. We can obtain the unconditional PEP from (32) by averaging it over $\mathbf{h}_{1}, \mathbf{h}_{2}$, and $\hat{\mathbf{g}}_{2}$. Unfortunately, it does not seem possible to derive a closed-form expression due to the presence of channel estimation errors. Hence, we will resort to numerical evaluation in Section $\mathrm{V}$ to investigate it. However, without channel estimation errors (i.e., $\phi_{e}^{2}=0$ ), the unconditional PEP can be derived in closed form as follows.

With $\phi_{e}^{2}=0$, we have

$$
\begin{aligned}
& \operatorname{Pr}\left(\mathbf{x}_{p} \rightarrow \hat{\mathbf{x}}_{p}, \mathbf{x}_{s} \rightarrow \hat{\mathbf{x}}_{s} \mid \mathbf{h}_{1}, \mathbf{h}_{2}, \mathbf{g}_{2}\right) \\
& =Q\left(\sqrt{\frac{1}{2 N_{0}} \sum_{\alpha=1}^{n} T(\alpha)}\right),
\end{aligned}
$$

where

$$
T(\alpha)=\frac{\left|A h_{1}(\alpha) h_{2}(\alpha) \Delta x_{p}(\alpha)+g_{2}(\alpha) \Delta x_{s}(\alpha)\right|^{2}}{1+A^{2}\left|h_{2}(\alpha)\right|^{2}}
$$

with $\Delta x_{p}(\alpha)=x_{p}(\alpha)-\hat{x}_{p}(\alpha)$ and $\Delta x_{s}(\alpha)=x_{s}(\alpha)-\hat{x}_{s}(\alpha)$. According to the following approximation of the $Q$-function:

$$
Q(x) \approx \frac{1}{12} \exp \left(-\frac{x^{2}}{2}\right)+\frac{1}{4} \exp \left(-\frac{2 x^{2}}{3}\right),
$$

(32) can be simplified as

$$
\begin{aligned}
& \operatorname{Pr}\left(\mathbf{x}_{p} \rightarrow \hat{\mathbf{x}}_{p}, \mathbf{x}_{s} \rightarrow \hat{\mathbf{x}}_{s} \mid \mathbf{h}_{1}, \mathbf{h}_{2}, \mathbf{g}_{2}\right) \\
& \approx \frac{1}{12} \prod_{\alpha=1}^{n} \exp \left(-q_{1} T(\alpha)\right)+\frac{1}{4} \prod_{\alpha=1}^{n} \exp \left(-q_{2} T(\alpha)\right),
\end{aligned}
$$

where $q_{1}=1 /\left(4 N_{0}\right)$ and $q_{2}=1 /\left(3 N_{0}\right)$. Thus, the unconditional PEP can be approximated as

$$
\begin{aligned}
& \operatorname{Pr}\left(\mathbf{x}_{p} \rightarrow \hat{\mathbf{x}}_{p}, \mathbf{x}_{s} \rightarrow \hat{\mathbf{x}}_{s}\right) \\
& \approx \frac{1}{12} \prod_{\alpha=1}^{n} E_{h_{1}, h_{2}, g_{2}}\left\{\exp \left(-q_{1} T(\alpha)\right)\right\} \\
& \quad+\frac{1}{4} \prod_{\alpha=1}^{n} E_{h_{1}, h_{2}, g_{2}}\left\{\exp \left(-q_{2} T(\alpha)\right)\right\} .
\end{aligned}
$$

For the calculation of $E_{h_{1}, h_{2}, g_{2}}\left\{\exp \left(-q_{i} T(\alpha)\right)\right\}, i=1,2$, we can consider four different scenarios, as described below.

- When $\Delta x_{p}(\alpha)=0$ and $\Delta x_{s}(\alpha)=0$, we have $T(\alpha)=0$ and $E_{h_{1}, h_{2}, g_{2}}\left\{\exp \left(-q_{i} T(\alpha)\right)\right\}=1$.
- When $\Delta x_{p}(\alpha)=0$ and $\Delta x_{s}(\alpha) \neq 0, T(\alpha)$ becomes

$$
T(\alpha)=\frac{\left|g_{2}(\alpha)\right|^{2}\left|\Delta x_{s}(\alpha)\right|^{2}}{1+A^{2}\left|h_{2}(\alpha)\right|^{2}} .
$$

Considering that $\left|g_{2}(\alpha)\right|^{2}$ and $\left|h_{2}(\alpha)\right|^{2}$ are mutually independent and exponentially distributed random variables with means equal to $\phi_{2}^{2}$ and $\sigma_{2}^{2}$, respectively, we arrive at

$$
\begin{aligned}
& E_{h_{1}, h_{2}, g_{2}}\left\{\exp \left(-q_{i} T(\alpha)\right)\right\} \\
& =E_{h_{2}}\left\{E_{g_{2} \mid h_{2}}\left\{\exp \left(-q_{i} T(\alpha)\right)\right\}\right\} \\
& =E_{h_{2}}\left\{\frac{1+A^{2}\left|h_{2}(\alpha)\right|^{2}}{1+A^{2}\left|h_{2}(\alpha)\right|^{2}+q_{i} \phi_{2}^{2}\left|\Delta x_{s}(\alpha)\right|^{2}}\right\} \\
& =\frac{1}{\sigma_{2}^{2}} \exp \left(\gamma / \sigma_{2}^{2}\right) \operatorname{Ei}\left(-\gamma / \sigma_{2}^{2}\right)\left(\gamma-1 / A^{2}\right)+1,
\end{aligned}
$$

where

$$
\gamma=\frac{1+q_{i} \phi_{2}^{2}\left|\Delta x_{s}(\alpha)\right|^{2}}{A^{2}} .
$$

- When $\Delta x_{p}(\alpha) \neq 0$ and $\Delta x_{s}(\alpha)=0, T(\alpha)$ becomes

$$
T(\alpha)=\frac{A^{2}\left|h_{1}(\alpha)\right|^{2}\left|h_{2}(\alpha)\right|^{2}\left|\Delta x_{p}(\alpha)\right|^{2}}{1+A^{2}\left|h_{2}(\alpha)\right|^{2}} .
$$

After some mathematical manipulations, we have

$$
\begin{aligned}
& E_{h_{1}, h_{2}, g_{2}}\left\{\exp \left(-q_{i} T(\alpha)\right)\right\} \\
& =E_{h_{2}}\left\{E_{h_{1} \mid h_{2}}\left\{\exp \left(-q_{i} T(\alpha)\right)\right\}\right\} \\
& =\frac{\exp \left(\gamma / \sigma_{2}^{2}\right) \operatorname{Ei}\left(-\gamma / \sigma_{2}^{2}\right)\left(A^{2} \gamma-1\right)+A^{2} \sigma_{2}^{2}}{A^{2} \sigma_{2}^{2}\left(1+q_{i} \sigma_{1}^{2}\left|\Delta x_{p}(\alpha)\right|^{2}\right)},
\end{aligned}
$$

where

$$
\gamma=\frac{1}{A^{2}\left(1+q_{i} \sigma_{1}^{2}\left|\Delta x_{p}(\alpha)\right|^{2}\right)} .
$$

- When $\Delta x_{p}(\alpha) \neq 0$ and $\Delta x_{s}(\alpha) \neq 0$, since $h_{1}(\alpha), h_{2}(\alpha)$, and $g_{2}(\alpha)$ are independent of each other, we have

$$
\begin{aligned}
& E_{h_{1}, h_{2}, g_{2}}\left\{\exp \left(-q_{i} T(\alpha)\right)\right\} \\
& =E_{h_{2}}\left\{E_{h_{1} \mid h_{2}}\left\{E_{g_{2} \mid h_{1}, h_{2}}\left\{\exp \left(-q_{i} T(\alpha)\right)\right\}\right\}\right\} .
\end{aligned}
$$

Given $h_{1}(\alpha)$ and $h_{2}(\alpha)$, we observe that $\left|A h_{1}(\alpha) h_{2}(\alpha) \Delta x_{p}(\alpha)+g_{2}(\alpha) \Delta x_{s}(\alpha)\right|^{2}$ is a noncentral chi-square random variable, whose moment generating function (MGF) is given by [32]

$$
\begin{aligned}
\mathcal{M}(t)= & \frac{1}{1-t \phi_{2}^{2}\left|\Delta x_{s}(\alpha)\right|^{2}} \\
& \times \exp \left(\frac{t\left|A h_{1}(\alpha) h_{2}(\alpha) \Delta x_{p}(\alpha)\right|^{2}}{1-t \phi_{2}^{2}\left|\Delta x_{s}(\alpha)\right|^{2}}\right) .
\end{aligned}
$$




$$
\begin{aligned}
& \operatorname{Pr}\left(\mathbf{x}_{p} \rightarrow \hat{\mathbf{x}}_{p}, \mathbf{x}_{s} \rightarrow \hat{\mathbf{x}}_{s} \mid \mathbf{h}_{1}, \mathbf{h}_{2}, \hat{\mathbf{g}}_{2}\right) \\
& =\operatorname{Pr}\left(-\sum_{\alpha=1}^{n}|x(\alpha)-\hat{x}(\alpha)|^{2}+2 \Re\left\{\bar{w}^{*}(\alpha)(x(\alpha)-\hat{x}(\alpha))\right\}>0\right) \\
& =Q\left(\frac{\sum_{\alpha=1}^{n}|x(\alpha)-\hat{x}(\alpha)|^{2}}{\sqrt{2 \phi_{e}^{2} \sum_{\alpha=1}^{n} r^{2}(\alpha)\left|x_{s}(\alpha)(x(\alpha)-\hat{x}(\alpha))\right|^{2}+2 N_{0} \sum_{\alpha=1}^{n}|x(\alpha)-\hat{x}(\alpha)|^{2}}}\right) .
\end{aligned}
$$

Hence, we obtain

$$
\begin{aligned}
& E_{g_{2} \mid h_{1}, h_{2}}\left\{\exp \left(-q_{i} T(\alpha)\right)\right\} \\
& =\frac{1+A^{2}\left|h_{2}(\alpha)\right|^{2}}{1+A^{2}\left|h_{2}(\alpha)\right|^{2}+q_{i} \phi_{2}^{2}\left|\Delta x_{s}(\alpha)\right|^{2}} \\
& \quad \times \exp \left(-\frac{q_{i} A^{2}\left|h_{2}(\alpha)\right|^{2}\left|\Delta x_{p}(\alpha)\right|^{2}\left|h_{1}(\alpha)\right|^{2}}{1+A^{2}\left|h_{2}(\alpha)\right|^{2}+q_{i} \phi_{2}^{2}\left|\Delta x_{s}(\alpha)\right|^{2}}\right) .
\end{aligned}
$$

Since the MGF of $\left|h_{1}(\alpha)\right|^{2}$ is

$$
\mathcal{M}(t)=\frac{1}{1-t \sigma_{1}^{2}},
$$

using the MGF approach again yields (48), which is shown at the top of the next page. Finally, by averaging (48) over $\left|h_{2}(\alpha)\right|^{2}$, whose PDF is given by

$$
f(x)=\frac{1}{\sigma_{2}^{2}} \exp \left(-\frac{x}{\sigma_{2}^{2}}\right),
$$

we are led to [33, Eqs. (3.352-4, 3.353-5)]

$$
\begin{aligned}
& E_{h_{1}, h_{2}, g_{2}}\left\{\exp \left(-q_{i} T(\alpha)\right)\right\} \\
& =\frac{\exp \left(\gamma / \sigma_{2}^{2}\right) \operatorname{Ei}\left(-\gamma / \sigma_{2}^{2}\right)\left(A^{2} \gamma-1\right)+A^{2} \sigma_{2}^{2}}{A^{2} \sigma_{2}^{2}\left(1+q_{i} \sigma_{1}^{2}\left|\Delta x_{p}(\alpha)\right|^{2}\right)},
\end{aligned}
$$

where

$$
\gamma=\frac{1+q_{i} \phi_{2}^{2}\left|\Delta x_{s}(\alpha)\right|^{2}}{A^{2}\left(1+q_{i} \sigma_{1}^{2}\left|\Delta x_{p}(\alpha)\right|^{2}\right)} .
$$

To sum up, $E_{h_{1}, h_{2}, g_{2}}\left\{\exp \left(-q_{i} T(\alpha)\right)\right\}$ can be expressed as (52), shown at the top of the next page, where $\gamma$ is given by (51). By substituting (52) into (37), the closed-form unconditional PEP can be obtained. Having $\operatorname{Pr}\left(\mathbf{x}_{p} \rightarrow \hat{\mathbf{x}}_{p}, \mathbf{x}_{s} \rightarrow \hat{\mathbf{x}}_{s}\right)$, according to the well-known union bounding technique, $P_{I}$ can be upper bounded by

$$
P_{I} \leq \frac{1}{2^{p_{1}+p_{2}+p_{3}}} \sum_{\mathbf{x}_{p}, \mathbf{x}_{s}} \sum_{\substack{\hat{\mathbf{x}}_{p}, \hat{\mathbf{x}}_{s} \\ \mathcal{I} \neq \hat{\mathcal{I}}}} \operatorname{Pr}\left(\mathbf{x}_{p} \rightarrow \hat{\mathbf{x}}_{p}, \mathbf{x}_{s} \rightarrow \hat{\mathbf{x}}_{s}\right) .
$$

\section{B. Calculation of $P_{s b}$}

Provided that the SAP is detected correctly, the instantaneous end-to-end SNR per subcarrier for the PU can be written as

$$
\rho_{u}=\frac{\frac{\left|h_{1}\right|^{2}}{N_{0}} \frac{\left|h_{2}\right|^{2}}{N_{0}}}{\frac{\left|h_{2}\right|^{2}}{N_{0}}+\frac{1}{A^{2} N_{0}}}=\frac{\rho_{1} \rho_{2}}{\rho_{2}+Z},
$$

where $\rho_{1}=\left|h_{1}\right|^{2} / N_{0}, \rho_{2}=\left|h_{2}\right|^{2} / N_{0}$, and $Z=1 /\left(A^{2} N_{0}\right)$. The MGF of $\rho_{u}$ is derived in [29] as

$$
\begin{aligned}
\mathcal{M}_{\rho_{u}}(t)= & \frac{1}{1-\bar{\rho}_{1} t}+\frac{Z \bar{\rho}_{1} t}{\bar{\rho}_{2}\left(1-\bar{\rho}_{1} t\right)^{2}} \exp \left(\frac{Z}{\bar{\rho}_{2}\left(1-\bar{\rho}_{1} t\right)}\right) \\
& \times \operatorname{Ei}\left(-\frac{Z}{\bar{\rho}_{2}\left(1-\bar{\rho}_{1} t\right)}\right),
\end{aligned}
$$

where $\bar{\rho}_{1}=\rho n \sigma_{1}^{2} / k$ and $\bar{\rho}_{2}=\rho n \sigma_{2}^{2} / k$ denote the means of $\rho_{1}$ and $\rho_{2}$, respectively. On the other hand, the conditional BEP of $M$-ary demodulation can be expressed as [34]

$$
\begin{aligned}
P_{s b \mid \rho_{u}} \cong & \frac{2}{\max \left(\log _{2}(M), 2\right)} \\
& \times \sum_{i=1}^{\max (M / 4,1)} Q\left(\sqrt{2 \rho_{u} \sin ^{2}\left(\frac{(2 i-1) \pi}{M}\right)}\right)
\end{aligned}
$$

for PSK signaling and

$$
\begin{gathered}
P_{s b \mid \rho_{u}}=\frac{1}{\log _{2}(\sqrt{M})} \sum_{m=1}^{\log _{2}(\sqrt{M})} P_{s b \mid \rho_{u}}(m) \\
P_{s b \mid \rho_{u}}(m)=\frac{2}{\sqrt{M}} \sum_{i=0}^{\left(1-2^{-m}\right) \sqrt{M}-1}\left\{(-1)^{\left\lfloor\frac{i \cdot 2^{m-1}}{\sqrt{M}}\right\rfloor}\right. \\
\left.\times\left(2^{m-1}-\left\lfloor\frac{i \cdot 2^{m-1}}{\sqrt{M}}+\frac{1}{2}\right\rfloor\right) Q\left((2 i+1) \sqrt{\frac{3 \rho_{u}}{M-1}}\right)\right\},
\end{gathered}
$$

for square QAM signaling. According to the approximation of the $Q$-function in (35) and the MGF approach, for $M$-ary PSK signaling, $P_{s b}$ can be derived as

$$
\begin{aligned}
P_{s b} \approx & \frac{2}{\max \left(\log _{2}(M), 2\right)} \\
\times \sum_{i=1}^{\max (M / 4,1)}\{ & \frac{1}{12} \mathcal{M}_{\rho_{u}}\left(-\sin ^{2}\left(\frac{(2 i-1) \pi}{M}\right)\right) \\
& \left.+\frac{1}{4} \mathcal{M}_{\rho_{u}}\left(-\frac{4}{3} \sin ^{2}\left(\frac{(2 i-1) \pi}{M}\right)\right)\right\},
\end{aligned}
$$




$$
E_{h_{1} \mid h_{2}}\left\{E_{g_{2} \mid h_{1}, h_{2}}\left\{\exp \left(-q_{i} T(\alpha)\right)\right\}\right\}=\frac{1+A^{2}\left|h_{2}(\alpha)\right|^{2}}{1+q_{i} \phi_{2}^{2}\left|\Delta x_{s}(\alpha)\right|^{2}+\left(1+q_{i} \sigma_{1}^{2}\left|\Delta x_{p}(\alpha)\right|^{2}\right) A^{2}\left|h_{2}(\alpha)\right|^{2}}
$$

$$
E_{h_{1}, h_{2}, g_{2}}\left\{\exp \left(-q_{i} T(\alpha)\right)\right\}= \begin{cases}1, & \text { for }\left(\Delta x_{p}(\alpha), \Delta x_{s}(\alpha)\right)=(0,0) \\ \frac{\exp \left(\gamma / \sigma_{2}^{2}\right) \operatorname{Ei}\left(-\gamma / \sigma_{2}^{2}\right)\left(A^{2} \gamma-1\right)+A^{2} \sigma_{2}^{2}}{A^{2} \sigma_{2}^{2}\left(1+q_{i} \sigma_{1}^{2}\left|\Delta x_{p}(\alpha)\right|^{2}\right)}, & \text { for }\left(\Delta x_{p}(\alpha), \Delta x_{s}(\alpha)\right) \neq(0,0),\end{cases}
$$

and for $M$-ary square QAM signaling, $P_{s b}$ is expressed as

$$
P_{s b}=\frac{1}{\log _{2}(\sqrt{M})} \sum_{m=1}^{\log _{2}(\sqrt{M})} P_{s b}(m)
$$

with

$$
\begin{aligned}
P_{s b \mid \rho_{u}}(m) \approx & \frac{2}{\sqrt{M}} \sum_{i=0}^{\left(1-2^{-m}\right) \sqrt{M}-1}\left\{(-1)^{\left\lfloor\frac{i \cdot 2^{m-1}}{\sqrt{M}}\right\rfloor}\right. \\
\times & \left(2^{m-1}-\left\lfloor\frac{i \cdot 2^{m-1}}{\sqrt{M}}+\frac{1}{2}\right\rfloor\right) \\
\times & {\left[\frac{1}{12} \mathcal{M}_{\rho_{u}}\left(-\frac{3(2 i+1)^{2}}{2(M-1)}\right)\right.} \\
& \left.\left.+\frac{1}{4} \mathcal{M}_{\rho_{u}}\left(-\frac{2(2 i+1)^{2}}{M-1}\right)\right]\right\} .
\end{aligned}
$$

Finally, substituting (53) and (59) (or (60)) into (26) yields the upper bound on the BER of the PU.

Remark 3: Similar to OFDM-IM, in OFDM-IM-CR with $\phi_{e}^{2}=0$, the IM bits have higher protection due to second order diversity, while the ordinary modulation bits have only one-diversity-order protection [22]. At high SNR, $P_{I}$ is very small compared with $P_{s b}$. Therefore, the BEP of OFDM-IM$\mathrm{CR}$ in (26) can be asymptotically approximated by

$$
P_{u} \approx \frac{p_{2} P_{s b}}{p_{1}+p_{2}}, \quad \rho \rightarrow \infty
$$

\section{Simulation Results}

In this section, we perform Monte Carlo simulations to evaluate the uncoded BER performance of the PU at the PR for the proposed OFDM-IM-CR scheme in the single-PU/SU scenario, where the BER of the PU is defined as the ratio of the number of bits in error at PR to the total number of transmitted bits from the PT. To examine the impact of the involvement of the SU on the PU, the OFDM-IM-AF scheme with the optimal ML detection, which is directly derived by removing the secondary network from OFDM-IM$\mathrm{CR}$, is taken as a benchmark for comparison. The OFDM$\mathrm{CR}$ scheme that is inspired from [35] is also chosen as a reference. In OFDM-CR, the PT and ST employ classical OFDM, and the ST acts as an AF relay for the PU by superimposing the primary and secondary signals with power allocation factors $\xi$ and $1-\xi$, respectively. The $\mathrm{PT} \rightarrow \mathrm{ST}$ link is set to follow frequency-selective Rician fading with the

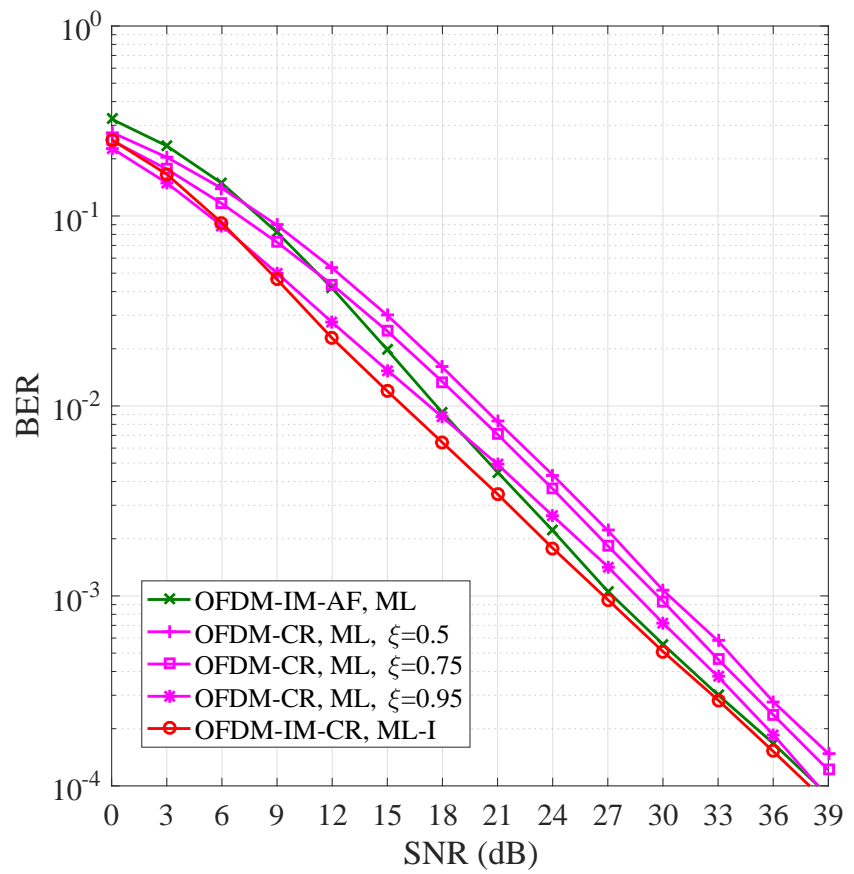

Fig. 2. Performance comparison among OFDM-IM-AF, OFDM-CR, and OFDM-IM-CR with $n=2, k=1, \sigma_{1}^{2}=\sigma_{2}^{2}=\phi_{2}^{2}=1, \phi_{e}^{2}=0$, and BPSK.

Rician factor $K=5$, which corresponds to the scenario where the PT and ST are located close together. In all simulations, BER curves are plotted versus $\rho, P_{S}=P_{R}$, and each BER point is obtained by averaging over at least ten times $1 / \mathrm{BER}$ samples for sufficient convergence. It is assumed that perfect time-frequency synchronization is achieved and the CSI of the $\mathrm{PT} \rightarrow \mathrm{R}$ and $\mathrm{R} \rightarrow \mathrm{PR}$ links is perfectly known at the PR. For brevity, we refer to the first type of near-ML/ML detector as "near-ML/ML-I", and the second type as "near-ML/ML-II" for OFDM-IM-CR.

Fig. 2 presents the comparison results among OFDM-IMAF, OFDM-CR, and OFDM-IM-CR with $n=2, k=1, \sigma_{1}^{2}=$ $\sigma_{2}^{2}=\phi_{2}^{2}=1, \phi_{e}^{2}=0$, and binary PSK (BPSK), where OFDMIM-CR employs ML-I detection, and $\xi=0.5 / 0.75 / 0.95$ for OFDM-CR. Interestingly, since the involvement of the $\mathrm{SU}$ is able to enhance the reliability of SAP detection, it can be seen from Fig. 2 that OFDM-IM-CR outperforms OFDM-IM-AF in the low-to-medium SNR region, while they almost obtain the same BER performance in the high SNR 


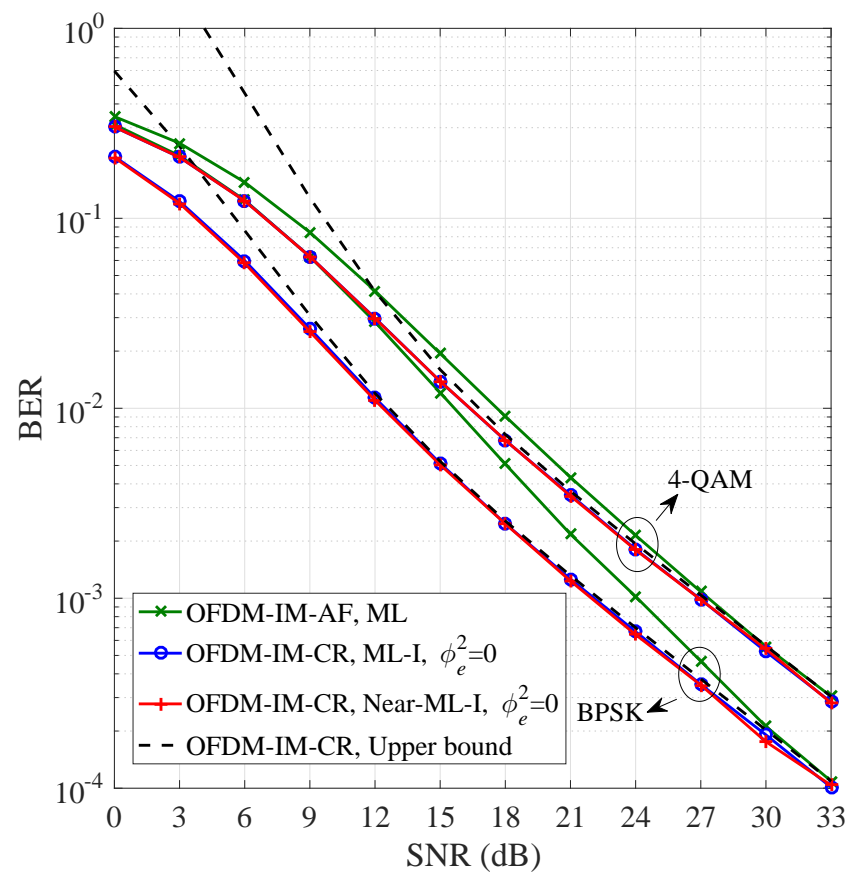

Fig. 3. Performance comparison between OFDM-IM-AF and OFDM-IM-CR with $n=4, k=1, \sigma_{1}^{2}=\sigma_{2}^{2}=\phi_{2}^{2}=1, \phi_{e}^{2}=0$, and BPSK/4-QAM.

region. For OFDM-CR, when $\xi$ tends to be larger, which means that the ST allocates more power to the primary signal, the BER performance of the PU improves. Note that, with increasing $\xi$, the performance of the $\mathrm{SU}$ is expected to be worse. Hence, choosing a proper value of $\xi$ that satisfies the quality of service requirement of the SU as well as improves the performance of the PU is a key issue in OFDM-CR. However, OFDM-IM-CR dispenses with power allocation, and it performs better than OFDM-CR in the SNR range of interest for $\xi=0.5 / 0.75 / 0.95$.

Fig. 3 presents the comparison results between OFDM-IM$\mathrm{AF}$ and OFDM-IM-CR with $n=4, k=1, \sigma_{1}^{2}=\sigma_{2}^{2}=$ $\phi_{2}^{2}=1, \phi_{e}^{2}=0$, and BPSK/4-QAM, where OFDM-IM-CR employs near-ML/ML-I detection. To verify the analysis given in Section IV, the BER upper bounds are also provided in Fig. 3 for OFDM-IM-CR. As seen from Fig. 3, for both BPSK and 4-QAM, the upper bounds agree with their computer simulation counterparts very well in the medium-to-high SNR region. The BER curves for the low-complexity near-ML-I detector almost overlap those for the optimal ML-I detector in the entire SNR region. Similar to the observations in Fig. 2, OFDM-IM-CR outperforms OFDM-IM-AF in the lowto-medium SNR region due to the higher reliability of SAP detection. Moreover, the gap between OFDM-IM-CR and OFDM-IM-AF for BPSK is larger than that for 4-QAM. This is because with higher modulation orders, OFDM-IM-CR has a greater error probability of SAP detection, while OFDM-IMAF almost keeps the same SAP detection performance [30]. In addition, for both OFDM-IM-AF and OFDM-IM-CR, an SNR loss of about $4 \mathrm{~dB}$ is observed from BPSK to 4-QAM, at a BER value of $10^{-3}$.

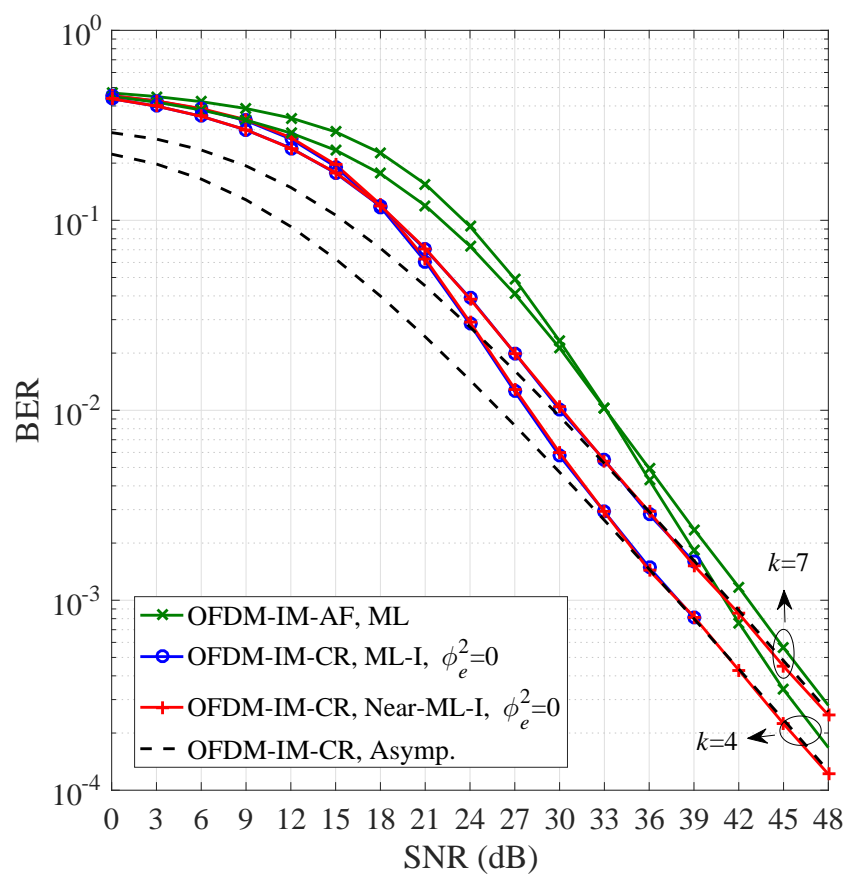

Fig. 4. Performance comparison between OFDM-IM-AF and OFDM-IM-CR with $n=8, k=4 / 7, \sigma_{1}^{2}=\sigma_{2}^{2}=\phi_{2}^{2}=1, \phi_{e}^{2}=0$, and 16-QAM.

Fig. 4 shows the comparison results when $n$ and $M$ increase to 8 and 16, respectively. The asymptotic expression in (62) is also given in Fig. 4 for OFDM-IM-CR. As seen from Fig. 4, (62) predicts the asymptotic behavior of OFDM-IMCR accurately. For both OFDM-IM-CR and OFDM-IM-AF, increasing $k$ from 4 to 7 results in worse asymptotic performance, which can be accounted for by the larger proportion of the constellation bits. Moreover, OFDM-IM-CR performs better than OFDM-IM-AF throughout the considered SNR region for both $k=4$ and $k=7$.

Fig. 5 shows the BER performance of the ML-I and nearML/ML-II detectors for OFDM-IM-CR, where $n=2, k=1$, $\phi_{e}^{2}=0 / 0.02 / 0.03$, and 4-QAM. As seen from Fig. 5, for the ML-I detector, the channel estimation errors degrade the performance greatly and result in error floors at high SNR. Due to the channel estimation errors and the nature of the union bound in (53), the BER upper bound for $\phi_{e}^{2}=0.02 / 0.03$ is looser than that for $\phi_{e}^{2}=0$. The low-complexity nearML-II detector performs the same as the optimal ML-II detector in the entire SNR region. When $\phi_{e}^{2}=0$, the nearML/ML-II detector performs worse than the ML-I detector for all considered SNR values. Interestingly, in the presence of channel estimation errors, although the ML-I detector is still superior to the ML-II detector at low SNR, the situation is reversed in the high SNR region. Moreover, the intersection lies at a smaller SNR value for a larger value of $\phi_{e}^{2}$.

Fig. 6 shows the BER curves of the ML-I and ML-II detectors for OFDM-IM-CR with $n=4, k=1 / 2 / 3, \phi_{e}^{2}=$ 0.03, and BPSK. As seen from Fig. 6, increasing $k$ results in worse performance for the ML-II detector in the overall SNR region since the value of $p_{2} /\left(p_{1}+p_{2}\right)$ becomes large. 


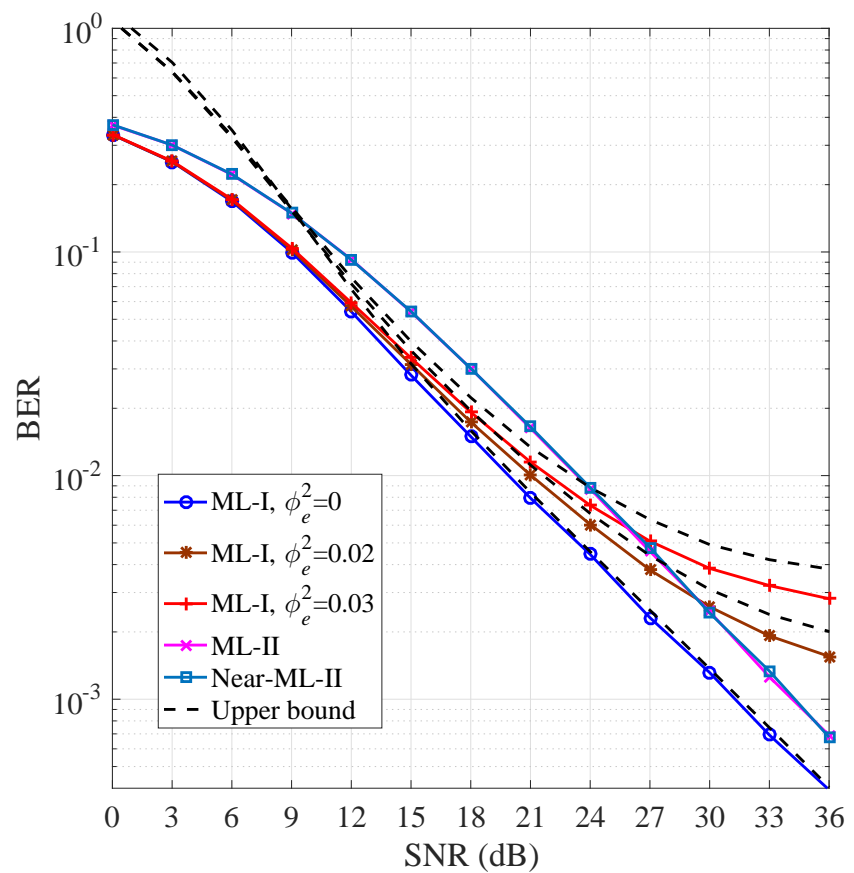

Fig. 5. Performance of the ML-I and ML-II detectors for OFDM-IM-CR with $n=2, k=1, \phi_{e}^{2}=0 / 0.02 / 0.03$, and 4-QAM.

However, this is not the case for the ML-I detector. Although a smaller value of $k$ leads to better performance at low SNR, it also generates higher error floors. This can be explained by (32), which indicates that the effect of channel estimation errors is highly influenced by the number of non-zero elements in $\mathbf{x}_{s}$. A smaller value of $k$ means that $\mathbf{x}_{s}$ has more non-zero elements, thus incurring higher error floors.

Fig. 7 gives the comparison results between the BER performance of the ML-I and ML-II detectors for OFDM-IM-CR with $n=2, k=1, \phi_{e}^{2}=0$, and 4-QAM, where four different link settings $\left(\sigma_{1}^{2}=\sigma_{2}^{2}=\phi_{2}^{2}=1 ; \sigma_{1}^{2}=10, \sigma_{2}^{2}=\phi_{2}^{2}=1\right.$; $\sigma_{1}^{2}=1, \sigma_{2}^{2}=10, \phi_{2}^{2}=1 ; \sigma_{1}^{2}=\sigma_{2}^{2}=1, \phi_{2}^{2}=10$ ) are considered. As shown in Fig. 5, increasing $\sigma_{1}^{2} / \sigma_{2}^{2} / \phi_{2}^{2}$ enhances the BER performance of both types of ML detectors. In particular, increasing $\sigma_{2}^{2}$ produces the greatest improvement in the SNR region of interest for both the ML-I and ML-II detectors. Since increasing $\phi_{2}^{2}$ contributes only to the SAP detection and has no effect on the $M$-ary symbol demodulation for the ML-I detector, the BER curves for $\phi_{2}^{2}=1$ and $\phi_{2}^{2}=10$ gradually approach one another with increasing SNR.

\section{CONCLUSIONS AND REMARKS}

In this paper, we have proposed OFDM-IM-CR for spectrum sharing, in which the PU adopts AF aided OFDM-IM for transmission, while the SU modulates its information on those inactive subcarriers of the PU. Two types of ML detectors have been designed for the PU, which are assisted by the estimated CSI and the statistical channel information of the secondary network, respectively. A complexity-reducing method has been proposed for both detectors, resulting in two types of lowcomplexity near-ML detectors. BER analysis has been provid-

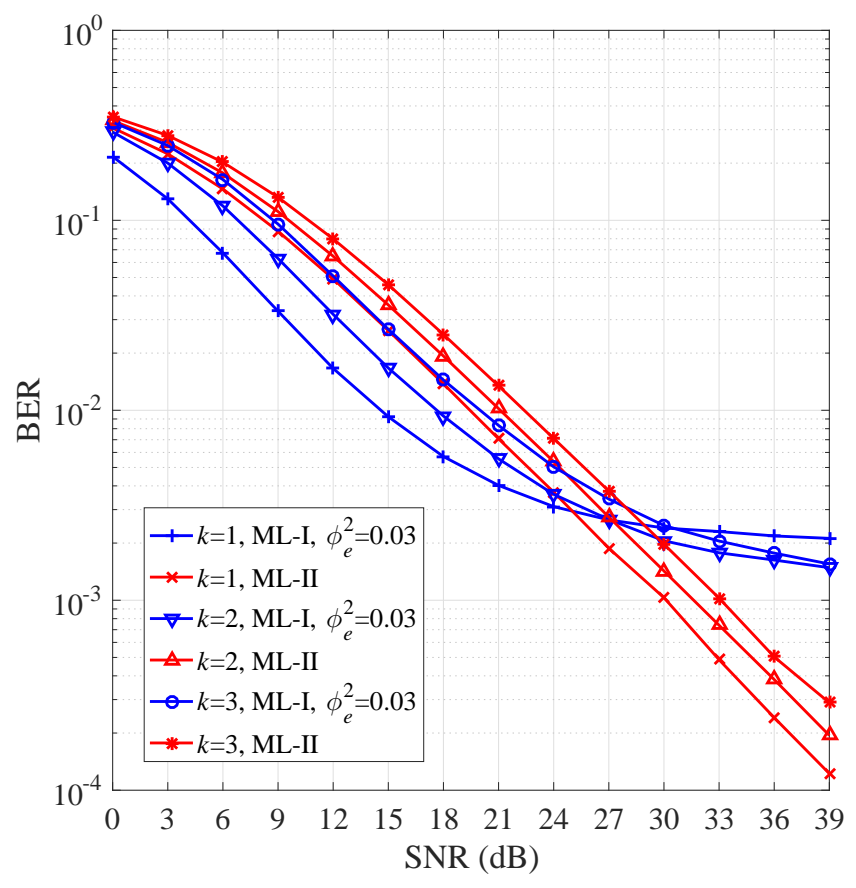

Fig. 6. Performance of the ML-I and ML-II detectors for OFDM-IM-CR with $n=4, k=1 / 2 / 3, \phi_{e}^{2}=0.03$, and BPSK.

ed assuming the first type of ML detection. In addition to the improvement of spectral efficiency, computer simulations have shown that the performance of the PU can be enhanced with the involvement of the SU. OFDM-IM-CR provides significant opportunities in, but not limited to, wireless sensor networks [36], wireless local area networks [37], and television networks [38] for spectrum sharing.

We remark that OFDM-IM-CR can be extended to the multi-antenna scenario in which all nodes are equipped with multiple antennas. More specifically, similar to multiple-input multiple-output (MIMO-)OFDM-IM [39], [40], the OFDM-IM principle is carried out simultaneously in each antenna of the PT. However, different from MIMO-OFDM-IM, multi-antenna OFDM-IM-CR assigns the same IM bit stream to all antennas of the PT per transmission. At the ST, after obtaining the indices of inactive subcarriers, each antenna simultaneously transmits constellation symbols over those inactive subcarriers. As a final comment, note that we have excluded the detrimental impacts of high peak-to-average power ratio, imperfect channel estimation of $\mathbf{h}_{1}$ and $\mathbf{h}_{2}$, and imperfect time-frequency synchronization in order to focus on the potential of OFDMIM in the exploration of the inherently inactive subcarriers for spectrum sharing. However, these are issues of interest for future study.

\section{REFERENCES}

[1] E. Axell, G. Leus, E. G. Larsson, and H. V. Poor, "Spectrum sensing for cognitive radio: State-of-the-art and recent advances," IEEE Signal Process. Mag., vol. 29, no. 3, pp. 101-116, May 2012.

[2] J. Mitola and G. Q. Maguire, "Cognitive radio: Making software radios more personal," IEEE Pers. Commun., vol. 6, no. 4, pp. 13-18, Aug. 1999. 


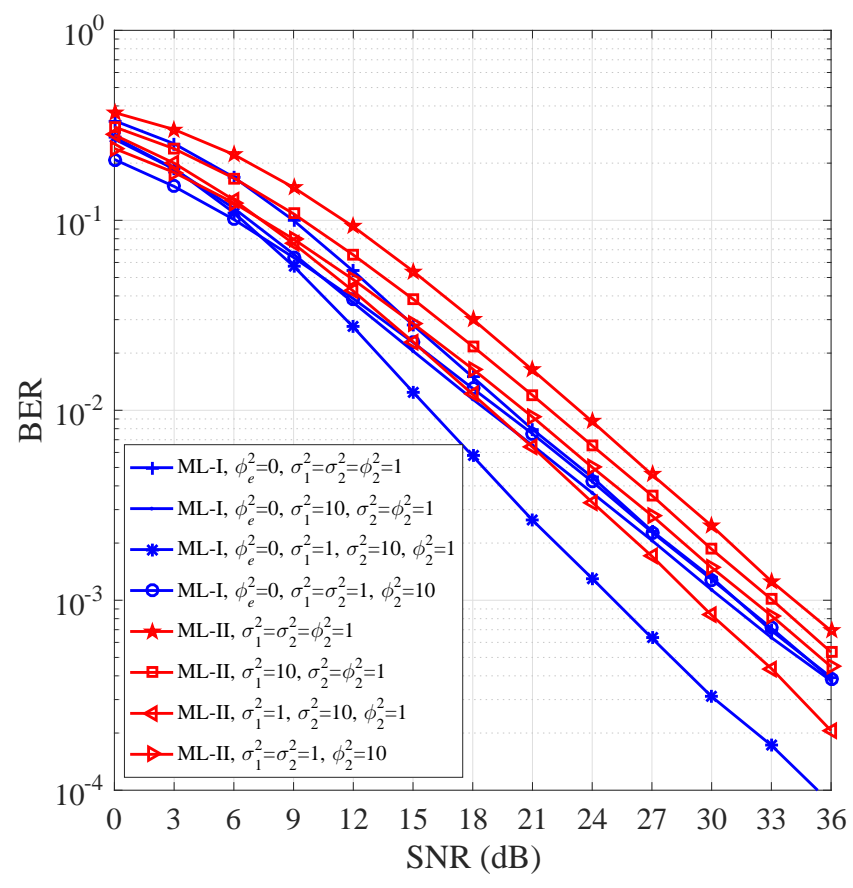

Fig. 7. Performance of the ML-I and ML-II detectors for OFDM-IM-CR with $n=2, k=1, \phi_{e}^{2}=0$, and 4-QAM, where four different link setups are considered.

[3] S. Haykin, "Cognitive radio: Brain-empowered wireless communications," IEEE J. Select. Areas Commun., vol. 23, no. 2, pp. 201-220, Feb. 2005.

[4] L. B. Le and E. Hossain, "Resource allocation for spectrum underlay in cognitive radio networks," IEEE Trans. Wireless Commun., vol. 7, no. 12 , pp. 5306-5315, Dec. 2008.

[5] G. Ganesan and $\mathrm{Y}$. Li, "Cooperative spectrum sensing in cognitive radio part I: Two user networks," IEEE Trans. Wireless Commun., vol. 6, no. 6, pp. 2204-2213, Jun. 2007.

[6] T. Yucek and H. Arslan, "A survey of spectrum sensing algorithms for cognitive radio applications," IEEE Commun. Surv. \& Tut., vol. 11, no. 1, pp. 116-130, 1st Quart., 2009.

[7] Y. Zeng and Y.-C. Liang, "Eigenvalue-based spectrum sensing algorithms for cognitive radio," IEEE Trans. Commun., vol. 57, no. 6, pp. 1784-1793, Jun. 2009.

[8] S. Haykin, D. J. Thomson, and J. H. Reed, "Spectrum sensing for cognitive radio," Proc. IEEE, vol. 97, no. 5, pp. 849-877, May 2009.

[9] H. A. Mahmoud, T. Yucek, and H. Arslan, "OFDM for cognitive radio: Merits and challenges," IEEE Wireless Commun., vol. 16, no. 2, pp. 615, Apr. 2009.

[10] T. A. Weiss and F. K. Jondral, "Spectrum pooling: An innovative strategy for the enhancement of spectrum efficiency," IEEE Commun. Mag., vol. 42, no. 3, pp. S8-S14, Mar. 2004.

[11] Y. Zhang and C. Leung, "Resource allocation in an OFDM-based cognitive radio system," IEEE Trans. Commun., vol. 57, no. 7, pp. 19281931, Jul. 2009.

[12] G. Bansal, M. Hossain, and V. Bhargava, "Optimal and suboptimal power allocation schemes for OFDM-based cognitive radio systems," IEEE Trans. Wireless Commun., vol. 7, no. 11, pp. 4710-4718, Nov. 2008.

[13] S. Chaudhari, V. Koivunen, and H. Vincent Poor, "Autocorrelation-based decentralized sequential detection of OFDM signals in cognitive radios," IEEE Trans. Signal Process., vol. 57, no. 7, pp. 2690-2700, Jul. 2009.

[14] A. Ali and W. Hamouda, "Spectrum monitoring using energy ratio algorithm for OFDM-based cognitive radio networks," IEEE Trans. Wireless Commun., vol. 14, no. 4, pp. 2257-2268, Apr. 2015.

[15] W. D. Lu, Y. Gong, S. H. Ting, X. L. Wu, and N. T. Zhang, "Cooperative OFDM relaying for opportunistic spectrum sharing: Protocol design and resource allocation," IEEE Trans. Wireless Commun., vol. 11, no. 6, pp. 2126-2135, Jun. 2012.

[16] E. Basar, U. Aygolu, E. Panayirci, and H. V. Poor, "Orthogonal frequen- cy division multiplexing with index modulation," IEEE Trans. Signal Process., vol. 61, no. 22, pp. 5536-5549, Nov. 2013.

[17] M. Wen, X. Cheng, and L. Yang, Index Modulation for $5 G$ Wireless Communications. Berlin, Germany: Springer, 2017.

[18] X. Cheng, M. Zhang, M. Wen, and L. Yang, "Index modulation for 5G: Striving to do more with less," IEEE Wireless Commun. Mag., vol. 25, no. 2, pp. 126-132, Apr. 2018.

[19] E. Basar, M. Wen, R. Mesleh, M. Di Renzo, Y. Xiao, and H. Haas, "Index modulation techniques for next-generation wireless networks," IEEE Access, vol. 5, pp. 16693-16746, Sep. 2017.

[20] M. Di Renzo, H. Haas, A. Ghrayeb, S. Sugiura, and L. Hanzo, "Spatial modulation for generalized MIMO: Challenges, opportunities and implementation," Proc. IEEE, vol. 102, no. 1, pp. 56-103, Jan. 2014.

[21] Q. Li, M. Wen, E. Basar, and F. Chen, "Index modulated OFDM spread spectrum," IEEE Trans. Wireless Commun., vol. 17, no. 4, pp. 23602374, Apr. 2018.

[22] M. Wen, B. Ye, E. Basar, Q. Li, and F. Ji, "Enhanced orthogonal frequency division multiplexing with index modulation," IEEE Trans. Wireless Commun., vol. 16, no. 7, pp. 4786-4801, Jul. 2017.

[23] S. Dang, J. P. Coon, and G. Chen, "Adaptive OFDM with index modulation for two-hop relay-assisted networks," IEEE Trans. Wireless Commun., vol. 17, no. 3, pp. 1923-1936, Mar. 2018.

[24] J. Crawford and Y. Ko, "Cooperative OFDM-IM relay networks with partial relay selection under imperfect CSI," IEEE Trans. Veh. Technol., vol. 67 , no. 10 , pp. $9362-9369$, Oct. 2018.

[25] S. Dang, G. Chen, and J. P. Coon, "Outage performance of two-hop OFDM with index modulation and multi-carrier relay selections," IEEE Wireless Commun. Lett., vol. 7, no. 6, pp. 926-929, Dec. 2018.

[26] G. Sheng, S. Dang, Z. Zhang, E. Kocan, and M. Pejanovic-Djurisic, "OFDM with index modulation assisted by multiple amplify-andforward relays," IEEE Wireless Commun. Lett., vol. 8, no. 3, pp. 789792, Jun. 2019

[27] Z. Wang, S. Dang, and D. T. Kennedy, "Multi-hop index modulationaided OFDM with decode-and-forward relaying," IEEE Access, vol. 6, pp. 26457-26468, 2018.

[28] Q. Ma, P. Yang, L. Dan, X. He, Y. Xiao, and S. Li, "OFDM-IMaided cooperative relaying protocol for cognitive radio networks," in Proc. IEEE 18th Int. Workshop Signal Process. Adv. Wireless Commun. (SPAWC), Sapporo, Japan, Jul. 2017, pp. 1-5.

[29] M. O. Hasna and M.-S. Alouini, "A performance study of dual-hop transmissions with fixed gain relays," IEEE Trans. Wireless Commun., vol. 3, no. 6, pp. 1963-1968, Nov. 2004.

[30] T. V. Luong and Y. Ko, "Impact of CSI uncertainty on MCIK-OFDM: Tight closed-form symbol error probability analysis," IEEE Trans. Veh. Technol., vol. 67, no. 2, pp. 1272-1279, Feb. 2018.

[31] T. V. Luong and Y. Ko, "A tight bound on BER of MCIK-OFDM with greedy detection and imperfect CSI," IEEE Commun. Lett., vol. 21, no. 12, pp. 2594-2597, Dec. 2017.

[32] M. K. Simon, Probability Distributions Involving Gaussian Random Variables: A Handbook for Engineers and Scientists. Berlin, Germany: Springer, 2006.

[33] I. S. Gradshteyn and I. M. Ryzhik, Table of Integrals, Series, and Products, 7th ed., New York, NY, USA: Academic Press, 2007.

[34] M. K. Simon and M.-S. Alouini, Digital Communication Over Fading Channels, 2nd ed. New York, NY, USA: Wiley, 2005.

[35] Z. Chen and X.-D. Zhang, "Cooperative spectrum sharing based on amplify and forward relaying in cognitive radio networks," Wirel. Pers. Commun., vol. 67, no. 3, pp. 585-598, Aug. 2012.

[36] A. Ahmad, S. Ahmad, M. H. Rehmani, and N. U. Hassan, "A survey on radio resource allocation in cognitive radio sensor networks," IEEE Commun. Surv. \& Tut., vol. 17, no. 2, pp. 888-917, 2nd Quart., 2015.

[37] B. Dappuri and T. G. Venkatesh, "Design and performance analysis of multichannel MAC protocol for cognitive WLAN," IEEE Trans. Veh. Tech., vol. 67, no. 6, pp. 5317-5330, Jun. 2018.

[38] K. Illanko, M. Naeem, A. Anpalagan, and D. Androutsos, "Energyefficient frequency and power allocation for cognitive radios in television systems," IEEE Syst. J., vol. 10, no. 1, pp. 313-324, Mar. 2016.

[39] E. Basar, "On multiple-input multiple-output OFDM with index modulation for next generation wireless networks," IEEE Trans. Signal Process., vol. 64 , no. 15 , pp. $3868-3878$, Aug. 2016.

[40] B. Zheng, M. Wen, E. Basar, and F. Chen, "Multiple-input multipleoutput OFDM with index modulation: Low-complexity detector design," IEEE Trans. Signal Process., vol. 65, no. 11, pp. 2758-2772, Jun. 2017. 


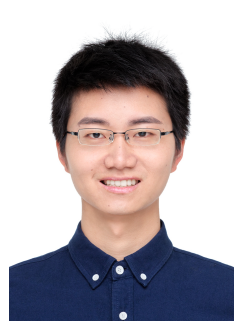

Qiang $\mathbf{L i}$ received the B.S. degree from Inner Mongolia University of Science and Technology, Baotou, China, in 2013, and the M.S. degree from Nanjing University of Aeronautics and Astronautics, Nanjing, China, in 2016. From 2018 to 2019, he was a Visiting Student Research Collaborator with Princeton University, Princeton, NJ, USA. He is pursuing the Ph.D. degree with South China University of Technology, Guangzhou, China. His recent research interests include index modulation and nonorthogonal multiple access.

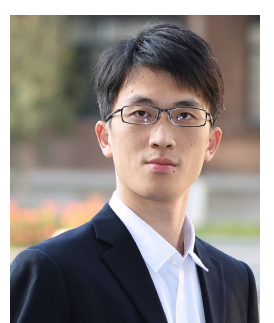

Miaowen Wen (M'14-SM'18) received the B.S. degree from Beijing Jiaotong University, Beijing, China, in 2009, and the Ph.D. degree from Peking University, Beijing, China, in 2014. From 2012 to 2013, he was a Visiting Student Research Collaborator with Princeton University, Princeton, NJ, USA. $\mathrm{He}$ is currently an Associate Professor with the South China University of Technology, Guangzhou, China. He has published a Springer book entitled Index Modulation for $5 G$ Wireless Communications and more than 70 journal papers. His research interests include index modulation, non-orthogonal multiple access, physical layer security, and molecular communications.

Dr. Wen was the recipient of the Excellent Doctoral Dissertation Award from Peking University and the Best Paper Awards from the IEEE ITST'12, the IEEE ITSC'14, and the IEEE ICNC'16. He was recognized as an Exemplary Reviewer for the IEEE COMMUNICATIONS LETTERS in 2017. He has served on the Editorial Boards of the IEEE ACCESS, and the EURASIP Journal on Wireless Communications and Networking, and a Guest Editor for IEEE Journal on Selected AREAS in Communications (Special Issue on Spatial Modulation for Emerging Wireless Systems) and for IEEE Journal of Selected Topics IN Signal Processing (Special Issue on Index Modulation for Future Wireless Networks: A Signal Processing Perspective). Currently, he is serving as an Editor for the IEEE TRANSACTIONS ON COMmunications, the IEEE COMmunications LetTERs, and the Physical Communication (Elsevier).

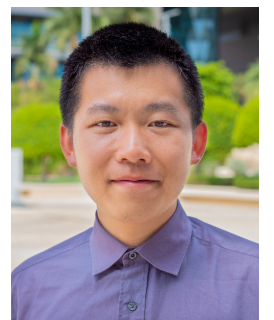

Shuping Dang (S'13-M'18) received B.Eng (Hons) in Electrical and Electronic Engineering from the University of Manchester (with first class honors) and B.Eng in Electrical Engineering and Automation from Beijing Jiaotong University in 2014 via a joint ' $2+2$ ' dual-degree program. He also received D.Phil in Engineering Science from University of Oxford in 2018. Dr. Dang joined in the R\&D Center, Huanan Communication Co., Ltd. after graduating from University of Oxford and is currently working as a Postdoctoral Fellow with the Computer, Electrical and Mathematical Science and Engineering Division, King Abdullah University of Science and Technology (KAUST). He serves as a reviewer for a number of key journals in communications and information science, including IEEE TRANSACTIONS ON WIRELESS COMMUNICATIONS, IEEE TRANSACTIONS on Communications, IEEE Wireless COMmunications LetTers, IEEE COMMUNICATIONS LETTERS, and IEEE TRANSACTIONS ON VEHICULAR TECHNOLOGY. His current research interests include novel modulation schemes, cooperative communications, and $6 \mathrm{G}$ wireless network design.

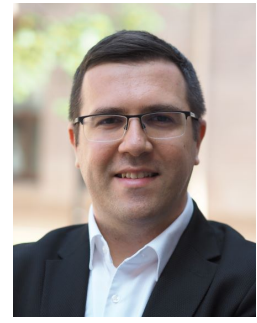

Ertugrul Basar (S'09-M'13-SM'16) received the B.S. degree (Hons.) from Istanbul University, Turkey, in 2007, and the M.S. and Ph.D. degrees from Istanbul Technical University, Turkey, in 2009 and 2013, respectively. From 2011 to 2012, he was with the Department of Electrical Engineering, Princeton University, Princeton, NJ, USA, as a Visiting Research Collaborator. He is currently an Associate Professor with the Department of Electrical and Electronics Engineering, Koç University, Istanbul, Turkey and the director of Communications Research and Innovation Laboratory (CoreLab). Previously, he was with the Department of Electronics and Communication Engineering, Istanbul Technical University. He is an inventor of five pending/granted patents on index modulation schemes. His primary research interests include MIMO systems, index modulation, intelligent surfaces, cooperative communications, OFDM, visible light communications, and signal processing for communications.

Recent recognition of his research includes the Science Academy (Turkey) Young Scientists (BAGEP) Award in 2018, Mustafa Parlar Foundation Research Encouragement Award in 2018, Turkish Academy of Sciences Outstanding Young Scientist (TUBA-GEBIP) Award in 2017, the first-ever IEEE Turkey Research Encouragement Award in 2017, and the Istanbul Technical University Best Ph.D. Thesis Award in 2014. He is also the recipient of five Best Paper Awards including one from the IEEE International Conference on Communications 2016. He has served as a TPC track chair or a TPC member for several IEEE conferences including GLOBECOM, VTC, PIMRC, and so on.

Dr. Basar currently serves as a Senior Editor of the IEEE COMMUNICATIONS LETTERS and the Editor of the IEEE TRANSACTIONS ON Communications and Physical Communication (Elsevier). In the past, he served as an Associate Editor for the IEEE COMMUNICATIONS LETTERS (2016-2019) and the IEEE ACCESS (2016-2018).

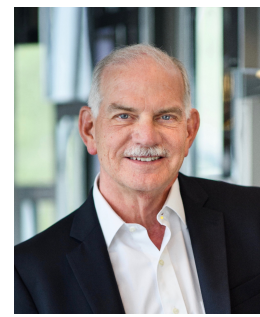

H. Vincent Poor (S'72-M'77-SM'82-F'87) received the Ph.D. degree in EECS from Princeton University in 1977. From 1977 until 1990, he was on the faculty of the University of Illinois at UrbanaChampaign. Since 1990 he has been on the faculty at Princeton, where he is currently the Michael Henry Strater University Professor of Electrical Engineering. During 2006 to 2016, he served as Dean of Princeton's School of Engineering and Applied Science. He has also held visiting appointments at several other universities, including most recently at Berkeley and Cambridge. His research interests are in the areas of information theory and signal processing, and their applications in wireless networks, energy systems and related fields. Among his publications in these areas is the recent book Multiple Access Techniques for $5 G$ Wireless Networks and Beyond. (Springer, 2019).

Dr. Poor is a member of the National Academy of Engineering and the National Academy of Sciences, and is a foreign member of the Chinese Academy of Sciences, the Royal Society, and other national and international academies. He received the Marconi and Armstrong Awards of the IEEE Communications Society in 2007 and 2009, respectively. Recent recognition of his work includes the 2017 IEEE Alexander Graham Bell Medal, the 2019 ASEE Benjamin Garver Lamme Award, a D.Sc. honoris causa from Syracuse University awarded in 2017, and a D.Eng. honoris causa from the University of Waterloo awarded in 2019. 


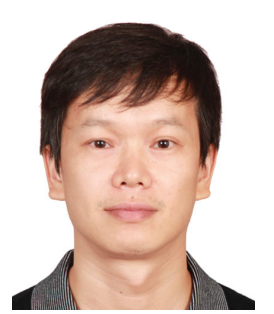

Fangiiong Chen (M'06) received the B.S. degree in electronics and information technology from Zhejiang University, Hangzhou, China, in 1997 and the $\mathrm{Ph} . \mathrm{D}$. degree in communication and information engineering from the South China University of Technology, Guangzhou, China, in 2002.

In 2002, he joined the School of Electronics and Information Engineering, South China University of Technology, where he was a Lecturer from 2002 to 2005, an Associate Professor from 2005 to 2011, and is currently a full-time Professor. He is also the Director of the Department of Underwater Detection and Imaging, Mobile Ultrasonic Detection National Research Center of Engineering Technology. His current research interests include signal detection and estimation, array signal processing, and wireless communication.

Dr. Chen was the recipient of the National Science Fund for Outstanding Young Scientists in 2013, and was elected in the New Century Excellent Talent Program of MOE, China, in 2012. 